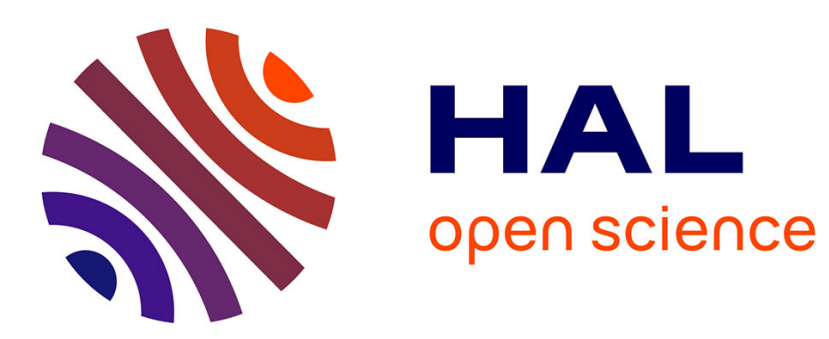

\title{
A trichotomy for regular simple path queries on graphs
}

\author{
Guillaume Bagan, Angela Bonifati, Benoit Groz
}

\section{To cite this version:}

Guillaume Bagan, Angela Bonifati, Benoit Groz. A trichotomy for regular simple path queries on graphs. Journal of Computer and System Sciences, 2020, 108, pp.29-48. 10.1016/j.jcss.2019.08.006 . hal-02435355

\section{HAL Id: hal-02435355 \\ https://hal.inria.fr/hal-02435355}

Submitted on 25 Sep 2020

HAL is a multi-disciplinary open access archive for the deposit and dissemination of scientific research documents, whether they are published or not. The documents may come from teaching and research institutions in France or abroad, or from public or private research centers.
L'archive ouverte pluridisciplinaire HAL, est destinée au dépôt et à la diffusion de documents scientifiques de niveau recherche, publiés ou non, émanant des établissements d'enseignement et de recherche français ou étrangers, des laboratoires publics ou privés. 


\title{
A trichotomy for regular simple path queries on graphs
}

\author{
Guillaume Bagan ${ }^{\mathrm{a}, *}$, Angela Bonifati ${ }^{\mathrm{a}}$, Benoit Groz ${ }^{\mathrm{b}}$ \\ a Université Lyon 1, LIRIS UMR CNRS 5205, F-69622, Lyon, France \\ ${ }^{\mathrm{b}}$ Université Paris Sud, LRI UMR CNRS 8623, F-91405, Orsay, France
}

\section{A R T I C L E I N F O}

\section{Article history:}

Received 3 June 2018

Received in revised form 23 June 2019

Accepted 19 August 2019

Available online 20 September 2019

\section{Keywords:}

Graphs

Paths

Regular simple paths

Complexity

Regular languages

Automata

\begin{abstract}
A B S T R A C T
We focus on the computational complexity of regular simple path queries (RSPQs). We consider the following problem $\mathrm{RSPQ}(\mathrm{L})$ for a regular language $L$ : given an edge-labeled digraph $G$ and two nodes $x$ and $y$, is there a simple path from $x$ to $y$ that forms a word belonging to $L$ ? We fully characterize the frontier between tractability and intractability for $\operatorname{RSPQ}(L)$. More precisely, we prove $\mathrm{RSPQ}(L)$ is either $\mathrm{AC}^{0}$, NL-complete or NP-complete depending on the language $L$. We also provide a simple characterization of the tractable fragment in terms of regular expressions. Finally, we also discuss the complexity of deciding whether a language $L$ belongs to the fragment above. We consider several alternative representations of $L$ : DFAs, NFAs or regular expressions, and prove that this problem is NL-complete for the first representation and PSPACE-complete for the other two.
\end{abstract}

(c) 2019 Elsevier Inc. All rights reserved.

\section{Introduction}

Graph databases have been investigated starting from the late 80s and are now again in vogue due to their wide application scenarios, ranging from social networks to biological and scientific databases (see [2] for a survey). Regular path queries (RPQs) are one of the most notable classes of queries on graph databases. They allow to retrieve pairs of nodes connected by a path, where the path is described through a regular expression. Such regular path queries are computable in time polynomial in both query and data complexity (combined complexity). In this paper, we investigate the computational complexity of regular simple path queries (RSPQs), a variant of RPQ in which the path connecting the pair has to be simple, i.e., does not have repeated vertices. Given an edge-labeled graph $G$ and a regular language $L$, an RSPQ selects pairs of vertices connected by a simple path whose edge labels form a word in $L$.

The evaluation of RSPQs is NP-complete even for fixed basic languages such as $(a a)^{*}$ or $a^{*} b a^{*}$ [20], in sharp contrast with RPQs. RSPQs are desirable in many application scenarios [17,23,6,15,13,30], such as transportation problems, VLSI design, metabolic networks, DNA matching and routing in wireless networks. Additionally, regular simple paths have been recently considered in SPARQL 1.1 queries exhibiting property paths. In particular, recent studies on the complexity of property paths in SPARQL $[3,18$ ] have highlighted the hardness of the semantics proposed by W3C to evaluate such paths in RDF graphs. Roughly speaking, according to the semantics considered in [18], the evaluation of expressions under Kleene-star closure should return a simple path, whereas the evaluation of the remaining expressions allows to traverse the same vertex multiple times. As such, the semantics studied in [18] is an hybrid between regular paths and regular simple paths semantics. RPQs have been recently found in practice within real-world SPARQL query logs, such as DBPedia and Wikidata

\footnotetext{
This article is an extended version of [5].

* Corresponding author.

E-mail addresses: guillaume.bagan@liris.cnrs.fr (G. Bagan), angela.bonifati@univ-lyon1.fr (A. Bonifati), benoit.groz@lri.fr (B. Groz).
} 
query logs $[8,7,9]$, showing thus the increasing interest of users manipulating real-world graph datasets with these queries. In particular, the majority of RPQs analyzed in large corpuses [8,9] belong to the tractable fragment $\mathcal{C}_{\text {tract }}$ studied in this paper, further confirming the applicability and impact of our theoretical investigation.

Contributions. In this paper, we address the long standing open question [20,6] of exactly characterizing the maximal class of regular languages for which RSPQs are tractable. By "tractable" we mean computable in time polynomial in the size of the graph. Precisely, we establish a comprehensive classification of the complexity of RSPQs for a fixed regular language $L$. A first step towards this important issue has been made in [20]. They exhibit a tractable fragment: the class of languages closed under taking subword. However, their fragment is not maximal.

Our contributions can be detailed as follows. We introduce a class of languages, named $\mathcal{C}_{\text {tract }}$, for which RSPQs can be evaluated in polynomial time (data complexity), and even in NL. We then show that RSPQ evaluation is NP-complete for every regular language that does not belong to $\mathcal{C}_{\text {tract }}$. Consequently, the maximal tractable fragment $\mathcal{C}_{\text {tract }}$ characterizes the frontier between tractability and intractability for this problem, under the hypothesis $\mathrm{NL} \neq \mathrm{NP}$. We further refine our results to show the following trichotomy: the evaluation of $\mathrm{RSPQ}$ problem is either $\mathrm{AC}^{0}$, NL-complete or NP-complete. We note that we consider the language $L$ as a fixed parameter, thus our results characterize the data complexity of RSPQ evaluation.

We also discuss the complexity of deciding, given a language $L$, whether the RSPQ problem for $L$ is tractable. We consider several alternative representations of $L$ : DFAs, NFAs or regular expressions. We prove that this problem of deciding tractability is NL-complete for the first representation and PSPACE-complete for the two others.

Next, we give a characterization of the tractable fragment $\mathcal{C}_{\text {tract }}$ for edge-labeled graphs in terms of regular expressions. We also show that $\mathcal{C}_{\text {tract }}$ is closed by union and intersection and show that languages in $\mathcal{C}_{\text {tract }}$ are aperiodic, i.e., can be expressed by first-order formulas [29].

We conclude with some minor results that identify further cases where RSPQs admit efficient solutions. We thus prove that RSPQs are FPT for the class of finite languages. Furthermore, we prove that the problem is also FPT for the class of all regular languages when the parameter is the size of the path. Finally, we prove that the problem RSPQ is polynomial w.r.t. combined complexity on graphs of bounded directed treewidth. This is actually a straightforward generalization of a result of [14].

A preliminary version of the present article has appeared in [5] without proofs of the main results. Here we provide detailed proofs.

Related work. A few papers deal with RSPQs or are related to them. Lapaugh et al. [16] prove that finding simple paths of even length is polynomial for non directed graphs and NP-complete for directed graphs. This study has been extended in [4] by considering paths of length $i$ mod $k$. Similarly, finding $k$ disjoint paths with extremities given as input is polynomial for non directed graphs [26] and NP-complete for directed graphs [10]. Using these results, Mendelzon and Wood prove that evaluating an RSPQ on an edge-labeled directed graph is NP-hard even for fixed languages [20]. However, they show that the problem can be decided in polynomial time for subword-closed languages. They also show that the problem becomes polynomial under some restrictions on the size of cycles of both graph and automaton. A subsequent paper [22] proves the polynomiality for the class of outerplanar graphs. Barrett et al. [6] extend this result, proving that the regular simple path problem is polynomial w.r.t. combined complexity for graphs of bounded treewidth. Barrett et al. [6] also show that the problem is NP-complete for the class of grid graphs even when the language is fixed.

We already mentioned that the complexity of evaluating SPARQL property paths has been investigated in previous studies $[18,3]$. As highlighted above, the semantics of SPARQL property paths blends the arbitrary paths and simple paths semantics. Losemann and Martens [18] and Arenas et al. [3] focus on the complexity of evaluating such property paths. They show that the evaluation is NP-complete in several cases, and exhibit cases in which it is polynomial. More precisely, Losemann and Martens [18] classify several fragments of property paths with respect to their complexity. Both the semantics and the query fragments are different from the ones in our paper. Counting the number of paths that match a regular expression (which is permitted for instance in SPARQL 1.1) is hard in many cases [18,3]. Recently, Martens and Trautner [19] studied the decision- and enumeration problems concerning the evaluation of RPQs by considering several semantics: arbitrary paths, shortest paths, and simple paths. While we prove here a trichotomy for the data complexity of the decision problem, they focus on the data complexity of the polynomial delay enumeration problem.

Section 2 introduces and illustrates the problem. We then establish our trichotomy. We first show in section 3 why languages outside our tractable fragment have NP-hard complexity. Section 4 discusses some properties of the tractable fragment, and shows how those properties (about strongly connected components) lead to a polynomial evaluation algorithm. And finally, Section 5 details our algorithm to deal with the tractable languages.

\section{Preliminaries}

Let $[n]$ denote the set of integers $\{1, \ldots, n\}$ and $[n, m]$ denote the set of integers $\{n, \ldots, m\}$.

\subsection{Complexity}

A TM refers to a Turing Machine and a NDTM refers to a non deterministic Turing Machine. $\mathrm{AC}^{0}, \mathrm{~L}, \mathrm{NL}, \mathrm{P}, \mathrm{NP}, \mathrm{PSPACE}$ refer to the classical classes of complexity [24]. The relations $\mathrm{AC}^{0} \subseteq \mathrm{L} \subset \mathrm{NL} \subseteq \mathrm{P} \subseteq \mathrm{NP} \subseteq$ PSPACE between these classes are well known. 
FL is the class of functions computable by a deterministic log-space transducer. The class $\mathrm{L}^{\mathrm{NL}}$ is the set of decision problems computable by a deterministic log-space algorithm with an oracle in NL. The class $\mathrm{FL}^{\mathrm{NL}}$ is the set of functions computable by a deterministic log-space transducer with an oracle in NL. The class $\mathrm{FL}^{\mathrm{NL}}(\mathrm{NL})$ is the set of problems reducible to a problem in NL by a function in $\mathrm{FL}^{\mathrm{NL}}$.

Lemma 1. [24,11] $\mathrm{NL}=\mathrm{L}^{\mathrm{NL}}=\mathrm{FL}^{\mathrm{NL}}(\mathrm{NL})$.

$\mathrm{NL}^{\mathrm{NL}}$ is the class of decision problems computable by a non deterministic log-space algorithm with an oracle in NL. The next lemma is true only if we make some restrictions on the oracle machine model (see [27]): the TM must write on the oracle tape deterministically i.e. it works deterministically as soon as it starts to write on the tape until it calls the oracle. The oracle tape is erased at the end of each call.

Lemma 2. [11] $\mathrm{NL}=\mathrm{NL}^{\mathrm{NL}}$.

\subsection{Graphs}

In our paper, we essentially consider db-graphs. A db-graph is a tuple $G=(V, \Sigma, E)$ where $V$ is a set of vertices, $\Sigma$ is a set of labels and $E \subseteq V \times \Sigma \times V$ is a set of edges labeled by symbols of $\Sigma$. Given a set $S \subseteq V$, G[S] the induced subgraph of $G$ by $S$ is $(S, \Sigma, E \cap S \times \Sigma \times S)$. A path $p$ of a db-graph $G$ from $x$ to $y$ is a sequence $\left(v_{1}=x, a_{1}, \ldots, v_{m}, a_{m}, v_{m+1}=y\right)$ such that for each $i \in[m+1], v_{i}$ is a vertex in $G$ and for each $i \in[m],\left(v_{i}, a_{i}, v_{i+1}\right)$ is an edge in $G$. A path $p$ is simple if all vertices $v_{i}$ in $p$ are distinct. Given a language $L \subseteq \Sigma^{*}, p$ is $L$-labeled if $a_{1} \ldots a_{m} \in L$. Given a subset $S \subseteq V, p$ is $S$-restricted if every intermediate vertex of $p$ belongs to $S$. Given a simple path $p$ and two vertices $x$ and $y$ in $p, p[x, y]$ denotes the subpath of $p$ from $x$ to $y$.

\subsection{Languages and automata}

Let $L$ be a regular language. Given a word $w$ and a language $L, w^{-1} L=\left\{w^{\prime}: w w^{\prime} \in L\right\}$. We denote by $A_{L}=$ $\left(Q_{L}, i_{L}, F_{L}, \Delta_{L}\right)$ the minimal DFA for $L$, and by $M_{L}$ the number of states $M_{L}=\left|Q_{L}\right|$ in $A_{L}$. Whenever the language is obvious from context, we drop the subscript and write $M$ instead of $M_{L}$. We assume that $A_{L}$ is complete i.e. $\Delta_{L}$ is a total function, so that in general $A_{L}$ may have a sink state. For any state $q \in Q$ and word $w \in \Sigma^{*}, \Delta_{L}(q, w)$ denotes the state obtained when reading $w$ from $q$. For any state $q \in Q$ and set of words $S \subseteq \Sigma^{*}, \Delta_{L}(q, S)$ denotes the set of states $q^{\prime}$ such that there exists $w \in S$ with $q^{\prime}=\Delta_{L}(q, w)$. Finally, $\mathscr{L}_{q}$ denotes the set of all words accepted from $q$. For every state $q$ we denote by $\operatorname{Loop}(q)$ the set of all non empty words that allow to loop on $q$ : $\operatorname{Loop}(q)=\left\{w \in \Sigma^{+} \mid \Delta_{L}(q, w)=q\right\}$. We say that a state $q^{\prime}$ is reachable from a state $q$ if $q^{\prime} \in \Delta_{L}\left(q, \Sigma^{*}\right)$. A (strongly connected) component of $A_{L}$ is a maximal set of states that are pairwise reachable.

The run of $L$ (or $A_{L}$ ) over a path $p=\left(v_{1}, a_{1}, \ldots, a_{m}, v_{m+1}\right)$ is the mapping $\rho:\left\{v_{1}, \ldots, v_{m+1}\right\} \rightarrow Q_{L}$ such that: $\rho\left(v_{1}\right)=i_{L}$ and $\rho\left(v_{i+1}\right)=\Delta_{L}\left(i_{L}, a_{1} \ldots a_{i}\right)$ for every $i \in[m]$. There are many characterizations of aperiodic languages [29]. A language $L$ is aperiodic if and only if it satisfies $\Delta_{L}\left(q, w^{M+1}\right)=\Delta_{L}\left(q, w^{M}\right)$ for every state $q$ and word $w$. Intuitively, that means that for any state $q_{0}$ and a word $w$, in the infinite sequence $\left(q_{0}, q_{1}, q_{2}, \ldots\right)$ with $q_{i+1}=\Delta_{L}(q, w)$ for any integer $i$, there is an integer $M$ such that $q^{M+1}=q^{M}$.

\subsection{Regular simple paths}

Given a regular language $L$, we define the following problem:

\section{$\operatorname{RSPQ}(L)$}

Input: A db-graph $G=(V, \Sigma, E)$, and two vertices $x, y \in V$

Question: Is there a simple $L$-labeled path from $x$ to $y$ ?

For this problem, $L$ is fixed, so we focus on data complexity. Notice that the representation of $L$ does not matter here. Although we consider the Boolean version of the problem, namely deciding the existence of a path, our algorithms actually also return a simple $L$-labeled path.

The main problem that we address in this paper is to distinguish cases when $\operatorname{RSPQ}(L)$ is tractable (i.e. decidable in polynomial time) and when it is not (i.e. NP-hard).

\subsection{The class of tractable languages}

We recall that $M$ refers to the size of $Q_{L}$, here and henceforth. We next introduce the class $\mathcal{C}_{\text {tract }}$ of languages. We will prove that it is exactly the class of regular languages for which $\operatorname{RSPQ}(L)$ is tractable. 
Definition 1. A regular language $L$ belongs to the class $\mathcal{C}_{\text {tract }}$ if the following property is satisfied: for all pairs of states $q_{1}, q_{2} \in Q_{L}$ and all words $w$ with $\operatorname{Loop}\left(q_{1}\right) \neq \emptyset, \operatorname{Loop}\left(q_{2}\right) \neq \emptyset, q_{2} \in \Delta_{L}\left(q_{1}, \Sigma^{*}\right)$ and $w \in \operatorname{Loop}\left(q_{2}\right)$, it holds that $w^{M} \mathscr{L}_{q_{2}} \subseteq \mathscr{L}_{q_{1}}$.

This definition is merely a technical definition for $\mathcal{C}_{\text {tract }}$, but we will provide in Theorem 6 a more intuitive characterizations of the class.

Example 1. As an introductory example, consider the language $L=a^{*}\left(b b^{+}+\epsilon\right) c^{*}$. We observe that this language belongs to our class $\mathcal{C}_{\text {tract }}$. We wish to decide RSPQ(L), i.e., whether there exists a simple path from $x$ to $y$ labeled by $L$, given two vertices $x, y$ of a db-graph $G$. It is not absolutely trivial that $\operatorname{RSPQ}(\mathrm{L})$ can be solved efficiently: $\operatorname{RSPQ}\left(a^{*} b c^{*}\right)$ has indeed been proved NP-complete. Yet we outline below a polynomial algorithm for $L$.

We distinguish two cases: there is a simple L-labeled path from $x$ to $y$ if and only if one of the following cases holds:

1: there exists a simple $a^{*} b^{k} c^{*}$-labeled path from $x$ to $y$ for some $k \in\{0,2,3\}$

2: case 1 does not hold and there exists a simple $a^{*} b^{4} b^{*} c^{*}$-labeled path from $x$ to $y$.

The first case is the easiest to check. We first check whether $y$ can be reached from $x$ by a (non-necessarily simple) $a^{*} c^{*}$-labeled path. If we find one, we obtain a simple $a^{*} c^{*}$-labeled path by eliminating its loops. Assume now there is no $a^{*} c^{*}$-labeled path from $x$ to $y$. We then check as follows if there exists a simple $a^{*} b^{k} c^{*}$-labeled path from $x$ to $y$ for some $k \in\{2,3\}$ : we try every possible assignment for the $k$ middle $b$-edges. For each combination of $k b$-edges, we check if the initial $b$-edge can be reached from $x$ through an $a^{*}$-labeled path (avoiding the vertices of the other $b$ edges), and check if the final $b$-edge can lead to $y$ through some $c^{*}$-labeled path (avoiding the vertices of the other $b$ edges). In the resulting $a^{*} b^{k} c^{*}$ labeled path the $a^{*}$-labeled prefix and $c^{*}$-labeled suffix cannot intersect (we assumed there is no $a^{*} c^{*}$ path). Consequently we obtain a simple $a^{*} b^{k} c^{*}$-labeled path by eliminating its loops. As the number of possible assignments for $k$ edges $(k \leq 3)$ is polynomial, we have proved that we can find out in polynomial time whether case 1 holds.

Let us now assume w.l.o.g. that there is no $a^{*} b^{k} c^{*}$-labeled path from $x$ to $y$ for $k \in\{0,2,3\}$. We can show that in this second case there exists a simple $L$-labeled path from $x$ to $y$ if and only if there exist six vertices $v_{1}, v_{2}, v_{3}, v_{4}, v_{5}, v_{6}$, two integers $l_{a}, l_{b}$ and two sets $S_{a}, S_{b}$ satisfying all following conditions:

- the vertices $v_{1}, \ldots, v_{6}$ are all distinct except that $v_{3}$ may equal $v_{4}$.

- there is a $b$-labeled edge from $v_{1}$ to $v_{2}$, from $v_{2}$ to $v_{3}$, from $v_{4}$ to $v_{5}$, and from $v_{5}$ to $v_{6}$.

- there is an $a^{*}$-labeled path from $x$ to $v_{1}$ avoiding all other $v_{i} \mathrm{~s}(i>1)$. The shortest possible such path has length $l_{a}$.

- $S_{a}$ is the set of all vertices reachable from $x$ through an $a^{*}$-labeled path of length at most $l_{a}$ that avoids all $v_{i} \mathrm{~s}(i>1)$.

- there is a $b^{*}$-labeled path from $v_{3}$ to $v_{4}$ of which all vertices (but the first and last) avoid $S_{a}$ and the $v_{i}$ s. The shortest possible such path has length $l_{b}$.

- $S_{b}$ is the set of all vertices reachable from $v_{3}$ through any $b^{*}$-labeled path of length at most $l_{b}$ that avoids $S_{a}$ and all other $v_{i} \mathrm{~s}(i \neq 4)$.

- there is a $c^{*}$-labeled path from $v_{6}$ to $y$ of which all vertices (but the first) avoid $S_{a}$ and $S_{b}$ and all other $v_{i} s(i<6)$.

The figure below summarizes all these conditions.

$$
\begin{aligned}
& x \stackrel{a^{*}}{\longrightarrow} v_{1} \stackrel{b}{\longrightarrow} v_{2} \stackrel{b}{\longrightarrow} v_{3} \stackrel{b^{*}}{\longrightarrow} v_{4} \stackrel{b}{\longrightarrow} v_{5} \stackrel{b}{\longrightarrow} v_{6} \stackrel{c^{*}}{\longrightarrow} y \\
& S_{a} \quad S_{b} \subseteq V \backslash S_{a} \quad \subseteq V \backslash\left(S_{a} \cup S_{b}\right)
\end{aligned}
$$

These conditions can clearly be verified in time polynomial in $G$. It is relatively clear also that the path constructed above is an $L$-labeled simple path from $x$ to $y$, so the conditions are sufficient to obtain an $L$-labeled simple path. To prove that our procedure is correct, we only have to prove that reciprocally, if there exists a simple $L$-labeled path we can find one satisfying our restrictions (the conditions above involving the $v_{i}, S_{a}, S_{b}$ ).

For every shortest $L$-labeled simple path $p$ from $x$ to $y$, let $v_{1}, \ldots, v_{6}$ denote the vertices that delimit the first and last two $b$-edges of $p$. We next show that those vertices satisfy the conditions above. The last vertex of $p$ that belongs to $S_{a}$ cannot occur after $v_{3}$ in $p$. Otherwise, we could obtain a simple path $p^{\prime}$ by replacing the prefix of $p$ up to $v$ with a shorter path through $S_{a}$. This resulting path $p^{\prime}$ would still be $L$-labeled by definition of the $v_{i}$, which contradicts the minimality of $p$. A similar argument shows that the last occurrence of a vertex from $S_{b}$ cannot occur after $v_{6}$. We conclude that the paths connecting $v_{3}$ to $v_{4}$ in $p$ (resp. $v_{6}$ to $y$ ) exclude respectively all vertices from $S_{a}$ (resp. $S_{a} \cup S_{b}$ ). As a consequence, the path from $x$ to $v_{1}$ will only feature vertices from $S_{a}$ by minimality of $p$, which proves that vertices $v_{1}, \ldots, v_{6}$ satisfy the conditions above.

The crux of our approach is to construct the $a^{*}, b^{*}$ and $c^{*}$ subpaths independently, lest we enumerate exponentially many paths. This is why we require that the $b^{*}$ subpath avoids $S_{a}$ : this condition is stronger than necessary to guarantee the first two subpaths do not intersect, but the stronger requirement allows us to build the two subpaths independently, as $S_{a}$ is a superset of the vertices on the subpath from $x$ to $v_{1}$. Our algorithm for tractable instances will generalize this idea. 


\section{Hard languages for RSPQ}

This section is devoted to the proof of a hardness result: $\operatorname{RSPQ}(L)$ is NP-hard for every regular language $L$ that does not belong to $\mathcal{C}_{\text {tract }}$. The first step toward that proof lies in the following characterization of $\mathcal{C}_{\text {tract }}$.

Definition 2 (Witness of hardness). Let $L$ be a regular language. A witness for hardness of $L$ is a tuple $\left(w_{l}, w_{m}, w_{r}, w_{1}, w_{2}\right)$ where $w_{l}, w_{r} \in \Sigma^{*}$ and $w_{m}, w_{1}, w_{2} \in \Sigma^{+}$satisfying

- $w_{l} w_{1}^{*} w_{m} w_{2}^{*} w_{r} \subseteq L$

- $w_{l}\left(w_{1}+w_{2}\right)^{*} w_{r} \cap L=\emptyset$.

Lemma 3. Let $L$ be a regular language that does not belong to $\mathcal{C}_{\text {tract. }}$ Then, $L$ admits a witness for hardness.

Proof. Let $L$ be a regular language that does not belong to $\mathcal{C}_{\text {tract }}$. For commodity, we distinguish two cases, depending on whether $L$ satisfies or not the following property: $\mathscr{L}_{q_{2}} \subseteq \mathscr{L}_{q_{1}}$ for every $q_{1}, q_{2} \in Q_{L}$ such that $q_{2} \in \Delta_{L}\left(q_{1}, \Sigma^{*}\right)$ and $\operatorname{Loop}\left(q_{1}\right) \cap \operatorname{Loop}\left(q_{2}\right) \neq \emptyset$ (Property $\mathcal{P}$ ).

Let $L$ be a language that does not satisfy Property $\mathcal{P}$, there exist $q, q_{2}, w_{m}, w, w_{r}$ such that $\Delta_{L}\left(q, w_{m}\right)=q_{2}, w \in L o o p(q) \cap$ $\operatorname{Loop}\left(q_{2}\right)$, and $w_{r} \in \mathscr{L}_{q_{2}} \backslash \mathscr{L}_{q}$. Let $w_{l}$ such that $\Delta\left(i_{L}, w_{l}\right)=q$. Then $w_{l}, w_{m}, w_{r}, w_{1}=w_{2}=w$ is a witness for hardness.

We next plan to exhibit a witness for hardness for the case where $L$ satisfies Property $\mathcal{P}$, but we first prove that every language satisfying property $\mathcal{P}$ (whether in $\mathcal{C}_{\text {tract }}$ or not) is aperiodic. Let $L$ be a language satisfying Property $\mathcal{P}, q \in Q_{L}$ and $w$ a word in $\Sigma^{+}$. Let also $q^{\prime}$ denote the state $q^{\prime}=\Delta_{L}\left(q, w^{M}\right)$. We denote by $q^{\prime \prime}$ the state $\Delta_{L}\left(q^{\prime}, w\right)$. We want to prove that $q^{\prime}=q^{\prime \prime}$. By the pigeonhole principle there exists some $k_{0}<k_{1} \leq M$ such that $\Delta_{L}\left(q, w^{k_{0}}\right)=\Delta_{L}\left(q, w^{k_{1}}\right)$. We then have $\Delta_{L}\left(q^{\prime}, w^{k}\right)=q^{\prime}$ for $k=k_{1}-k_{0}$. Then $q^{\prime}$ and $q^{\prime \prime}$ both loop on $w^{k}$, so that $\mathscr{L}_{q^{\prime}}=\mathscr{L}_{q^{\prime \prime}}$ by definition of $\mathcal{P}$, hence $q^{\prime}=q^{\prime \prime}$ by minimality. Consequently, $L$ is aperiodic.

Let $L$ be a language that satisfies Property $\mathcal{P}$ (and so in particular is aperiodic), but that does not belong to $\mathcal{C}_{\text {tract }}$. By definition of $\mathcal{C}_{\text {tract }}$ there exist states $q, q_{2}$ and words $w_{l}, w_{1}, w_{2}, w_{m}, w_{r}^{\prime}$ such that $\Delta\left(i_{L}, w_{l}\right)=q, w_{1} \in \operatorname{Loop}(q), w_{2} \in$ $\operatorname{Loop}\left(q_{2}\right), \Delta_{L}\left(q, w_{m}\right)=q_{2}, w_{r}^{\prime} \in \mathscr{L}_{q_{2}}$ and $w_{2}^{M} w_{r}^{\prime} \notin \mathscr{L}_{q}$. W.l.o.g. we can suppose that $w_{1}=\left(w_{1}^{\prime}\right)^{M}$ for some word $w_{1}^{\prime}$. We then claim that $\mathscr{L}_{q^{\prime}} \subseteq \mathscr{L}_{q}$ for every $q^{\prime}$ in $\Delta_{L}\left(q, \Sigma^{*} w_{1}\right)$. Indeed, for every $q^{\prime} \in \Delta_{L}\left(q, \Sigma^{*} w_{1}\right)$, there exists some $k>0$ such that $\Delta_{L}\left(q^{\prime}, w_{1}^{k}\right)=q^{\prime}$, hence $q^{\prime}$ loops over $w_{1}$ by aperiodicity of $L$. We thus have $w_{1} \in \operatorname{Loop}(q) \cap \operatorname{Loop}\left(q^{\prime}\right)$ and therefore $\mathscr{L}_{q^{\prime}} \subseteq \mathscr{L}_{q}$ due to Property $\mathcal{P}$.

Let $w_{r}=w_{2}^{M} w_{r}^{\prime}$. By definition, $w_{m} w_{2}^{*} w_{r} \subseteq \mathscr{L}_{q}$ because $w_{r} \in \mathscr{L}_{q_{2}}$. We now prove that $\left(w_{1}+w_{2}\right)^{*} w_{r} \cap \mathscr{L}_{q}=\emptyset$, because any word in $\left(w_{1}+w_{2}\right)^{*} w_{r}$ can be decomposed into $u v$ with $u \in \epsilon+\left(w_{1}+w_{2}\right)^{*} w_{1}$ and $v \in\left(w_{2}\right)^{*} w_{r}$. We recall that $w_{r}=w_{2}^{M} w_{r}^{\prime} \notin \mathscr{L}_{q}$ and $L$ is aperiodic, so that $v \notin \mathscr{L}_{q}$. Furthermore, we have just proved that $q^{\prime}=\Delta_{L}(q, u)$ satisfies $\mathscr{L}_{q} \subseteq \mathscr{L}_{q}$. Consequently, $v \notin \mathscr{L}_{q^{\prime}}$ and $u v \notin \mathscr{L}_{q}$. Thus, $w_{l}, w_{m}, w_{r}, w_{1}$, and $w_{2}$ provide a witness for hardness, which concludes the proof of Lemma 3.

We can now prove our hardness result, by reduction from Vertex-Disjoint-Path, a problem also used in [20] to prove hardness in the particular case of $a^{*} b a^{*}$.

\section{Vertex-Disjoint-Path}

Input: A directed graph $G=(V, E)$, four vertices $x_{1}, y_{1}, x_{2}, y_{2} \in V$

Question: Are there two disjoint paths, one from $x_{1}$ to $y_{1}$ and the other from $x_{2}$ to $y_{2}$ ?

Lemma 4. Let $L$ be a regular language that does not belong to $\mathcal{C}_{\text {tract }}$. Then, $R S P Q(L)$ is NP-hard.

Proof. Let $L \notin \mathcal{C}_{\text {tract }}$. We exhibit a reduction from the Vertex-Disjoint-Path problem to RSPQ $(L)$. According to Lemma 3, $L$ admits a witness for hardness $w_{l}, w_{m}, w_{r}, w_{1}, w_{2}$. By definition we get $w_{l}\left(w_{1}+w_{2}\right)^{*} w_{r} \cap L=\emptyset$ and $w_{l} w_{1}^{*} w_{m} w_{2}^{*} w_{r} \subseteq L$.

We build from $G$ a db-graph $G^{\prime}$ whose edges are labeled by non empty words. This is actually a generalization of dbgraphs. Nevertheless, by adding intermediate vertices, an edge labeled by a word $w$ can be replaced with a path whose edges form the word $w$.

$G^{\prime}$ is constructed as follows. The vertices of $G^{\prime}$ are the same as the vertices of $G$. For each edge $\left(v_{1}, v_{2}\right)$ in $G$, we add two edges $\left(v_{1}, w_{1}, v_{2}\right)$ and $\left(v_{1}, w_{2}, v_{2}\right)$. Moreover, we add two new vertices $x, y$ and three edges $\left(x, w_{l}, x_{1}\right),\left(y_{1}, w_{m}, x_{2}\right)$ and $\left(y_{2}, w_{r}, y\right)$. We next prove that $\operatorname{RSPQ}(L)$ returns True for $\left(G^{\prime}, x, y\right)$ iff Vertex-Disjoint-Path returns True for $\left(G, x_{1}, y_{1}, x_{2}, y_{2}\right)$.

Assume there is a simple $L$-labeled path $p$ from $x$ to $y$ in $G^{\prime}$. By definition of $G^{\prime}$, this path necessarily goes through the edge $\left(y_{1}, w_{m}, x_{2}\right)$ since $w_{l}\left(w_{1}+w_{2}\right)^{*} w_{r} \cap L=\emptyset$. Since $p$ is simple, the subpaths from $x_{1}$ to $y_{1}$ and $x_{2}$ to $y_{2}$ are disjoint, hence Vertex-Disjoint-Path returns True for $\left(G, x_{1}, y_{1}, x_{2}, y_{2}\right)$. Reciprocally, if Vertex-Disjoint-Path returns True for $\left(G, x_{1}, y_{1}, x_{2}, y_{2}\right)$, there exist disjoint paths from $x_{1}$ to $y_{1}$ and from $x_{2}$ to $y_{2}$. By definition these two paths match a word in $\left(w_{1}+w_{2}\right)^{*}$. We can then obtain two disjoint simple paths, one from $x_{1}$ to $y_{1}$ matching a word in $w_{1}^{*}$ and one from $x_{2}$ to $y_{2}$ matching a word in $w_{2}^{*}$. To obtain those paths we keep the vertices as the original paths, eliminate the loops if 


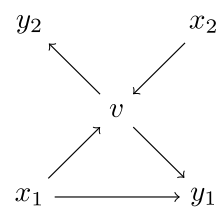

Input instance $G$

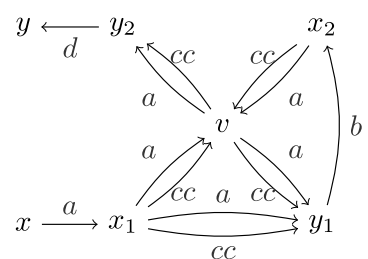

RSPQ instance: graph $G^{\prime}$

Fig. 1. Reduction for $L=a^{*} b(c c)^{*} d$

there are any, and switch $w_{1}$ and $w_{2}$ edges where needed: we can always replace a $w_{1}$ edge with a $w_{2}$ by construction of $G$ since every pair of vertices is connected by both types of edges or none. Concatenating the edge $\left(x, w_{l}, x_{1}\right)$ with the first path, the edge $\left(y_{1}, w_{m}, x_{2}\right)$, the second path and the edge $\left(y_{2}, w_{r}, y\right)$ provides a simple $L$-labeled path $p$ from $x$ to $y$, which concludes our proof. We illustrate in Fig. 1 the reduction for $L=a^{*} b(c c)^{*} d$, on an instance $\left(G, x_{1}, y_{1}, x_{2}, y_{2}\right)$, choosing $w_{l}=w_{1}=a, w_{m}=b, w_{2}=c c$, and $w_{r}=d$.

This concludes our proof that languages outside $\mathcal{C}_{\text {tract }}$ are intractable. After this negative result, we now focus on the positive result, namely that languages in $\mathcal{C}_{\text {tract }}$ admit efficient algorithms.

\section{Properties of languages in $\mathcal{C}_{\text {tract }}$}

The main result of this paper is that for every $L \in \mathcal{C}_{\text {tract }}, \mathrm{RSPQ}(L) \in \mathrm{NL}$. The algorithm to evaluate efficiently $\operatorname{RSPQ}(L)$ exploits a particular kind of pumping argument between strongly connected components of the automaton. This pumping argument proves that if we build carefully a path using the usual reachability algorithm inside the strongly connected components, then we need not care about possible intersections between subpaths corresponding to different components. In this section, we introduce and prove this pumping argument in Lemma 11 through a serie of technical lemmas about the structure of automata that recognize $\mathcal{C}_{\text {tract }}$ languages.

\subsection{Alternative characterization of $\mathcal{C}_{\text {tract }}$}

To begin with, we prove that every language from $\mathcal{C}_{\text {tract }}$ is aperiodic and deduce an alternative characterization of $\mathcal{C}_{\text {tract }}$.

Lemma 5. Let $L$ be a regular language in $\mathcal{C}_{\text {tract }}$. Then $L$ is aperiodic.

Proof. In the proof of Lemma 3 we defined a property $\mathcal{P}$ and showed that languages satisfying property $\mathcal{P}$ are aperiodic. We show that every $L \in \mathcal{C}_{\text {tract }}$ satisfies property $\mathcal{P}$. Let $L \in \mathcal{C}_{\text {tract }}, q_{1}, q_{2} \in Q_{L}$ and $w$ satisfy $q_{2} \in \Delta_{L}\left(q_{1}, \Sigma^{*}\right)$ and $w \in \operatorname{Loop}\left(q_{1}\right) \cap$ Loop $\left(q_{2}\right)$. By definition of $\mathcal{C}_{\text {tract }}, w^{M} \mathscr{L}_{q_{2}} \subseteq \mathscr{L}_{q_{1}}$, hence $\mathscr{L}_{q_{2}} \subseteq \mathscr{L}_{q_{1}}$ because $w \in \operatorname{Loop}\left(q_{1}\right)$.

We then exploit this aperiodicity property to establish the following characterization of $\mathcal{C}_{\text {tract }}$, which strengthens the requirements from Definition 1 on the loops of $A_{L}$.

Lemma 6. Let $L$ be a regular language. Then, $L$ belongs to $\mathcal{C}_{\text {tract }}$ iff for every pair of states $q_{1}, q_{2} \in Q_{L}$ such that $\operatorname{Loop}\left(q_{1}\right) \neq \emptyset$, $\operatorname{Loop}\left(q_{2}\right) \neq \emptyset$ and $q_{2} \in \Delta_{L}\left(q_{1}, \Sigma^{*}\right)$, the following statement holds: $\left(\operatorname{Loop}\left(q_{2}\right)\right)^{M} \mathscr{L}_{q_{2}} \subseteq \mathscr{L}_{q_{1}}$.

Proof. The (if) implication is immediate by Definition 1. Let us now prove the (only if) implication. Assume $L \in \mathcal{C}_{\text {tract }}$. Let $q_{1}^{\prime}, q_{2}^{\prime} \in Q_{L}$ satisfy $\operatorname{Loop}\left(q_{1}^{\prime}\right) \neq \emptyset$, $\operatorname{Loop}\left(q_{2}^{\prime}\right) \neq \emptyset, q_{2}^{\prime} \in \Delta_{L}\left(q_{1}^{\prime}, \Sigma^{*}\right)$, and let $w \in \operatorname{Loop}\left(q_{2}^{\prime}\right)$. Let also $q_{3}$ denote the state $\Delta_{L}\left(q_{1}^{\prime}, w^{M}\right)$. Then Definition 1 implies $w^{M} \mathscr{L}_{q_{2}^{\prime}} \subseteq \mathscr{L}_{q_{1}^{\prime}}$. Thus, $\mathscr{L}_{q_{2}^{\prime}} \subseteq \mathscr{L}_{q_{3}}$. The crux of the proof is to choose carefully $q_{1}^{\prime}, q_{2}^{\prime}$ and $w$ to exploit the constraints on $\mathscr{L}_{q_{3}}$.

Let $q_{1}, q_{2}$ be two states such that $\operatorname{Loop}\left(q_{1}\right) \neq \emptyset$, $\operatorname{Loop}\left(q_{2}\right) \neq \emptyset$ and $q_{2} \in \Delta_{L}\left(q_{1}, \Sigma^{*}\right)$. Let $\left(v_{1}, \ldots, v_{M}\right)$ be a sequence of words in $\left(\operatorname{Loop}\left(q_{2}\right)\right)^{M}$ and $q_{3}=\Delta_{L}\left(q_{1}, v_{1} \ldots v_{M}\right)$. We wish to prove $\mathscr{L}_{q_{2}} \subseteq \mathscr{L}_{q_{3}}$.

For some $i, j, 0 \leq i<j \leq M$, we get $\Delta_{L}\left(q_{1}, v_{1} \ldots v_{i}\right)=\Delta_{L}\left(q_{1}, v_{1} \ldots v_{j}\right)$, using the convention $\Delta_{L}\left(q_{1}, v_{1} \ldots v_{i}\right)=q_{1}$ for $i=0$. Let $u_{1}=v_{1} \ldots v_{i}, u_{2}=v_{i+1} \ldots v_{j}$ and $u_{3}=v_{j+1} \ldots v_{M}$. Let $q_{4}=\Delta_{L}\left(q_{1}, u_{1}\right)$. We claim that $\mathscr{L}_{q_{2}} \subseteq \mathscr{L}_{q_{4}}$. The result then follows from $\mathscr{L}_{q_{2}}=u_{3}^{-1} \mathscr{L}_{q_{2}} \subseteq u_{3}^{-1} \mathscr{L}_{q_{4}}=\mathscr{L}_{q_{3}}$. To prove the claim, let $w=u_{1} u_{2}^{M}$ and $q_{5}=\Delta_{L}\left(q_{1}, w^{M}\right)$. As $\Delta_{L}\left(q_{1}, w^{M}\right)=$ $q_{5}$ and $w \in \operatorname{Loop}\left(q_{2}\right)$, we get $\mathscr{L}_{q_{2}} \subseteq \mathscr{L}_{q_{5}}$ through Definition 1 with $q_{1}, q_{2}$ and $w$. Furthermore, $u_{2}$ belongs to Loop $\left(q_{5}\right)$ because $L$ is aperiodic. To conclude the proof, we observe that $\mathscr{L}_{q_{5}} \subseteq \mathscr{L}_{q_{4}}$, by Definition 1 with $q_{5}, q_{4}$ and $u_{2}$, and because $\Delta_{L}\left(q_{4}, u_{2}^{M}\right)=q_{4}$ and $u_{2} \in \operatorname{Loop}\left(q_{5}\right){ }^{1}$

\footnotetext{
1 This last application of Definition 1 corresponds actually to observing that every language in $\mathcal{C}_{\text {tract }}$ satisfies property $\mathcal{P}$ from Lemma 3.
} 


\subsection{Technical lemmas on the components of $A_{L}$}

In this section, we show properties about the components of $\mathcal{C}_{\text {tract }}$ languages. Notice that states in a component are mutually reachable, but not reachable from states in other components that they can reach themselves. From now on, and until the end of the section, we fix a language $L \in \mathcal{C}_{\text {tract }}$. We introduce in Lemmas 9 and 11 the pumping argument that we exploit in the algorithm to compute a simple path. In the other lemmas we prove auxiliary results, based on the decomposition of the automaton in strongly connected components. We prove that components of languages in $\mathcal{C}_{\text {tract }}$ are very particular, in the sense that every word staying long enough in the component is synchronizing. A preliminary lemma shows that two distinct states $q_{1}$ and $q_{2}$ in the same component cannot loop on the same word.

Lemma 7. Let $q_{1}$ and $q_{2}$ be two states belonging to the same component of $A_{L}$. If $\operatorname{Loop}\left(q_{1}\right) \cap \operatorname{Loop}\left(q_{2}\right) \neq \emptyset$, then $q_{1}=q_{2}$.

Proof. Let $q_{1}, q_{2}$ as above, and let $w$ a word in $\operatorname{Loop}\left(q_{1}\right) \cap \operatorname{Loop}\left(q_{2}\right)$. According to Definition 1 , w $w^{M} \mathscr{L}_{q_{2}} \subseteq \mathscr{L}_{q_{1}}$, hence $\mathscr{L}_{q_{2}} \subseteq$ $\mathscr{L}_{q_{1}}$ since $w \in \operatorname{Loop}\left(q_{1}\right)$. By symmetry, $\mathscr{L}_{q_{2}}=\mathscr{L}_{q_{1}}$, which implies $q_{2}=q_{1}$.

The next two lemmas characterize the internal language of a component.

Lemma 8. Let $C$ be a component of $A_{L}, q_{1}, q_{2} \in C$ and $a \in \Sigma$. Then $\Delta_{L}\left(q_{1}, a\right) \in C$ iff $\Delta_{L}\left(q_{2}, a\right) \in C$.

Proof. Let $q_{1} \neq q_{2}$ two states in the same component $C$. Let $a$ satisfy $\Delta_{L}\left(q_{1}, a\right) \in C$. Let also $w \in \operatorname{Loop}\left(q_{1}\right) \cap a \Sigma^{*}$ and $q_{3}=\Delta_{L}\left(q_{2}, w^{M}\right): a$ and $w$ necessarily exist because $C$ is the strongly connected component of $q_{1}$ and $q_{2}$. We next prove that $q_{3}$ belongs to $C$ : by our definition of $C$, this implies $\Delta_{L}\left(q_{2}, a\right) \in C$. As $L$ is aperiodic, $w \in \operatorname{Loop}\left(q_{3}\right)$, and consequently, $w^{M} \mathscr{L}_{q_{3}} \subseteq \mathscr{L}_{q_{1}}$ by Definition 1. Furthermore, $w^{M} \mathscr{L}_{q_{1}} \subseteq \mathscr{L}_{q_{2}}$ also by Definition 1 . Hence $\mathscr{L}_{q_{3}} \subseteq \mathscr{L}_{q_{1}}$ and $\mathscr{L}_{q_{1}} \subseteq\left(w^{M}\right)^{-1} \mathscr{L}_{q_{2}}=$ $\mathscr{L}_{q_{3}}$. Thus, $\mathscr{L}_{q_{1}}=\mathscr{L}_{q_{3}}$ and, by minimality of $A_{L}, q_{1}=q_{3}$, so that $q_{3} \in C$.

Notation 1. We denote the internal alphabet of a component $C$ of $A_{L}$ by $\Sigma_{C}=\left\{a \in \Sigma: \exists q_{1}, q_{2} \in C . \Delta_{L}\left(q_{1}, a\right)=q_{2}\right\}$.

As a direct consequence of Lemma 8 we get:

Lemma 9. Let $C$ be a component of $A_{L}, q \in C$ and $w \in \Sigma^{*}$. Then $\Delta_{L}(q, w) \in C$ iff $w \in\left(\Sigma_{C}\right)^{*}$.

Finally, we prove that inside a component, every word with length at least $M^{2}$ is synchronizing. This result is the core of our pumping argument between strongly connected components as exposed in Lemma 11.

Lemma 10. Let $C$ be a component of $A_{L}, \Sigma_{C}$ be the internal alphabet of $C, q_{1}, q_{2}$ be two states of $C$ and $w \in\left(\Sigma_{C}\right)^{M^{2}}$. Then, $\Delta_{L}\left(q_{1}, w\right)=\Delta_{L}\left(q_{2}, w\right)$.

Proof. Assume that $w=a_{1} \ldots a_{M^{2}}$. For each $i$ from 0 to $M^{2}$ and $\alpha=1,2$, let $q_{\alpha, i}=\Delta_{L}\left(q_{\alpha}, a_{1} \ldots a_{i}\right)$. Since there are at most $M^{2}$ distinct pairs $\left(q_{1, i}, q_{2, i}\right)$, there exist $i, j$, with $i<j$ such that $q_{1, i}=q_{1, j}$ and $q_{2, i}=q_{2, j}$. By Lemma $9, q_{1, i}, q_{2, i} \in C$. Let $w^{\prime}=a_{i+1} \ldots a_{j}$. We have $w^{\prime} \in \operatorname{Loop}\left(q_{1, i}\right) \cap \operatorname{Loop}\left(q_{2, i}\right)$, hence $q_{1, i}=q_{2, i}$ by Lemma 7 . As a consequence, $\Delta_{L}\left(q_{1}, w\right)=$ $\Delta_{L}\left(q_{2}, w\right)$.

Notice that the above lemma still holds for $w \in\left(\Sigma_{C}\right)^{M^{2}} \Sigma_{C}^{*}$. Here and thereafter, we fix the constant $N=2 M^{2}$.

Lemma 11. Let $q_{1}, q_{2}$ be two states such that Loop $\left(q_{1}\right) \neq \emptyset$, Loop $\left(q_{2}\right) \neq \emptyset$, and $q_{2} \in \Delta_{L}\left(q_{1}, \Sigma^{*}\right)$. Let $C$ be the component that contains $q_{2}$ and $\Sigma_{C}$ be the internal alphabet of $C$. Then, $\mathscr{L}_{q_{2}} \cap\left(\Sigma_{C}\right)^{N} \Sigma^{*} \subseteq \mathscr{L}_{q_{1}}$.

Proof. Let $w \in \mathscr{L}_{q_{2}} \cap\left(\Sigma_{C}\right)^{N} \Sigma^{*}$. There are some words $u, v \in\left(\Sigma_{C}\right)^{M^{2}}, w^{\prime} \in \Sigma^{*}$ such that $w=u v w^{\prime}$. By Lemma 9 and the Pigeonhole Principle, there exist a state $q_{3} \in C$ and $M+1$ non-empty words $v_{1}, \ldots, v_{M+1}$ such that $v=v_{1} \ldots v_{M+1}$ and $\Delta_{L}\left(q_{2}, u v_{1} \ldots v_{i}\right)=q_{3}$ for every $i \in[M]$. Therefore, $w \in u v_{1}\left(\operatorname{Loop}\left(q_{3}\right)\right)^{M-1} v_{M+1} w^{\prime}$. By Lemma $10, \Delta_{L}\left(q_{3}, u v_{1}\right)=$ $\Delta_{L}\left(q_{2}, u v_{1}\right)=q_{3}$. Thus, $w$ belongs to both $\left(\operatorname{Loop}\left(q_{3}\right)\right)^{M} v_{M+1} w^{\prime}$ and $\mathscr{L}_{q_{3}}$. By Lemma $6, w \in \mathscr{L}_{q_{1}}$.

Or main result focuses on data complexity and therefore assumes the language (hence $N$ ) is constant. Yet the complexity will be exponential in $N$ therefore we next prove, for the sake of efficiency, that we can take $N=M$ in Lemma 11 .

Lemma 12. Let $q_{1}, q_{2}$ be two states such that $\operatorname{Loop}\left(q_{1}\right) \neq \emptyset$, Loop $\left(q_{2}\right) \neq \emptyset$, and $q_{2} \in \Delta_{L}\left(q_{1}, \Sigma^{*}\right)$. Let $C$ be the component that contains $q_{2}$ and $\Sigma_{C}$ be the internal alphabet of $C$. Then, $\mathscr{L}_{q_{2}} \cap\left(\Sigma_{C}\right)^{M} \Sigma^{*} \subseteq \mathscr{L}_{q_{1}}$. 
Proof. Lemma 10 shows that all words of length at least $M^{2}$ are synchronizing inside a component. A straightforward partition-refinement argument [25] shows that for every $k, k^{\prime}$ and every $k$-states DFA, if words of length $k^{\prime}$ are synchronizing, then words of length $k$ are already synchronizing. This shows that words of length $|C|$ are synchronizing inside a component of $A_{L}$, hence it is enough to assume $w \in\left(\Sigma_{C}\right)^{|C|}$ in Lemma 10.

With the assumptions of Lemma 11 , let $w=u v \in \mathscr{L}_{q_{2}}$ such that $u \in\left(\Sigma_{C}\right)^{M}$. As $u$ synchronizes $C, \Delta\left(q_{2}, u^{M+1} v\right)=$ $\Delta\left(q_{2}, u v\right) \in F$. If $\Delta\left(q_{1}, u\right) \in C$ then $\Delta\left(q_{1}, u^{M+1} v\right) \in F$ since $L \in \mathcal{C}_{\text {tract }}$. Furthermore, $\Delta\left(q_{1}, u v\right)=\Delta\left(q_{1}, u^{M+1} v\right)$ as $u$ synchronizes $C$. As a consequence, $\Delta\left(q_{1}, u v\right) \in F$.

Otherwise the automaton avoids $C$ while reading $u$ from $q_{1}$ and as a consequence there exist $C^{\prime}, u_{1}, u_{2}, u_{3}$ satisfying the following 4 conditions: $u=u_{1} u_{2} u_{3}, \Delta\left(q, u_{1}\right) \in C^{\prime}, u_{2} \in\left(\Sigma_{C^{\prime}}\right)^{\left|C^{\prime}\right|}$ and $\left|u_{3}\right| \geq|C|$. Then $\Delta\left(q_{2}, u_{1} u_{2}^{N} u_{3} v\right)=$ $\Delta\left(q_{2}, u v\right) \in F$ because $u_{3}$ synchronizes $C$. By Lemma 11 , this implies $\Delta\left(q_{1}, u_{1} u_{2}^{N} u_{3} v\right) \in F$. As $u_{2}$ synchronizes $C^{\prime}$, $\Delta\left(q_{1}, u v\right)=\Delta\left(q_{1}, u_{1} u_{2}^{N} u_{3} v\right)$ and therefore belongs to $F$. In both cases, $w \in \mathscr{L}_{q_{1}}$, which concludes our proof.

\section{Computing $\operatorname{RSPQ}(L)$ for $L$ in $\mathcal{C}_{\text {tract }}$}

In this section, we describe a polynomial algorithm that computes $\operatorname{RSPQ}(L)$ when $L$ belongs to $\mathcal{C}_{\text {tract }}$. A (non-necessarily simple) $L$-labeled path between two points could be computed incrementally using dynamic programming: we only need to record the last vertex in the (partial) path together with the corresponding state. But this approach is not adequate to build a simple path, as we need to memorize all the vertices in the path to check the absence of loops. The approach may thus lead to consider an exponential number of paths.

Nevertheless, we will show that in the case where $L$ belongs to $\mathcal{C}_{\text {tract }}$, we can identify a constant number of vertices that suffice to check if the path is (or can be transformed into) a simple path labeled with L. Section 5.1 defines path summaries that record these "critical" vertices, while Section 5.2 explains how we can restore any shortest simple $L$-labeled path from its summary. Finally, Section 5 presents our algorithm for RSPQ, which enumerates all candidate summaries in logarithmic space, until it finds a candidate summary from which it can restore a simple $L$-labeled path.

\subsection{Defining summaries}

Roughly speaking, the idea of a summary is to keep only a bounded number of vertices of $p$, that depends only on $L$. Notice that in a run of $A_{L}$ over $p$, states of the same component appear consecutively. Indeed, if one leaves a component of $A_{L}$, one cannot re-enter it later. Inspired by Lemma 12, we will only record the first and the $M$ last vertices having their state in $C$, for each component $C$ of $A_{L}$. When the number of such vertices is greater than $M+1$, we replace the path between the first vertex and the $M$ last ones by a cut symbol cut ${ }_{C}$. This symbol intuitively represents a $\Sigma_{C}^{*}$-labeled path that has been cut from the path. More formally, a summary is defined as follows.

Definition 3 (Long run components). Let $p=\left(v_{1}, a_{1}, \ldots, a_{m}, v_{m+1}\right)$ be a path and let $\rho$ be the run of $L$ over $p$. A long run component of $p$ is a component $C$ of $A_{L}$ such that there are at least $M+2$ vertices $v$ in $p$ such that $\rho(v) \in C$. We denote by $C_{1}, \ldots, C_{l}$ the long run components of $p$ (the sequence is sorted by order of appearance in $p$ ). For each integer $i \in[l]$, $\Sigma_{C_{i}}$ is the internal alphabet of $C_{i}$, left $t_{i}$ is the first vertex $v_{j}$ of $p$ such that $\rho\left(v_{j}\right) \in C_{i}$ and right $t_{i}$ is the last vertex $v_{j}$ of $p$ such that $\rho\left(v_{j}\right), \ldots, \rho\left(v_{j+M}\right) \in C_{i}$.

Definition 4 (Cut symbols and summary). We introduce a new "cut" symbol cut $_{C}$ for each component $C$ of $A_{L}$. The set of all cut symbols is denoted by Cuts. Let $p=\left(v_{1}, a_{1}, \ldots, a_{m}, v_{m+1}\right), \rho$ a run over $p$, and $\left(C_{i}, \Sigma_{C_{i}} \text {, left } t_{i} \text {, right }\right)_{i \in[l]}$ be as stated in Definition 3. The summary $S$ of the path $p$ (w.r.t. $A_{L}$ ) is the sequence obtained from $p$ by replacing, for each $i \in[l]$ the subpath $p\left[\right.$ left $_{i}$, right $\left._{i}\right]$ by the sequence $\left(\right.$ left $_{i}$, cut $_{C_{i}}$, right $\left.{ }_{i}\right)$.

Example 2 depicts a path together with its summary.

Example 2. Fig. 2a represents the minimal DFA for $L=a\left(c^{\geq 2}+\epsilon\right)(a+b)^{*}(a c) ? a^{*}$ (we did not represent the sink state). This automaton can loop in three strongly connected components: $C_{1}=\left\{q_{4}\right\}, C_{2}=\left\{q_{5}, q_{6}\right\}$, and $C_{3}=\left\{q_{7}\right\}$. The accepting states are $q_{2}, q_{4}, q_{5}, q_{6}$, and $q_{7}$. For this automaton, we observe that Lemma 12 still holds after replacing exponent $M$ with 2 . Consequently, we shall pretend that $M=2$ when defining the long run components and summary in our example. This means we only store the first and last 3 (instead of 8 ) vertices of each component, which will simplify our illustrative example.

Let us consider the path $p_{1}$ illustrated in Fig. $2 \mathrm{~b}$ with thick edges. Fig. $2 \mathrm{c}$ details the run over this path.

We observe that $p_{1}$ is a simple $L$-labeled path. The summary $S$ of $p_{1}$ is obtained by removing the second (resp. second and third) vertex with state in $C_{1}$ (resp. $C_{2}$ ). The vertices on a white background in Fig. $2 \mathrm{c}$ are thus eliminated from the summary and replaced with their respective cut symbol cut $C_{1}$ and $\mathrm{cut}_{C_{2}}$ :

$$
\begin{aligned}
S= & \left(v_{1}, a, v_{2}, c, v_{3}, c, v_{4}, \text { cut }_{C_{1}}, v_{7}, c, v_{8}, c, v_{9},\right. \\
& \left.a, v_{10}, \text { cut }_{C_{2}}, v_{13}, a, v_{14}, a, v_{15}\right) .
\end{aligned}
$$




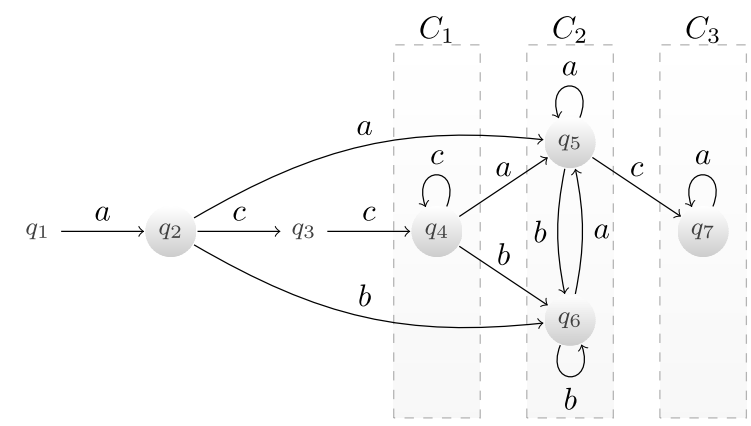

(a) Minimal DFA for $L=a\left(c^{\geq 2}+\epsilon\right)(a+b)^{*}(a c) ? a^{*}$

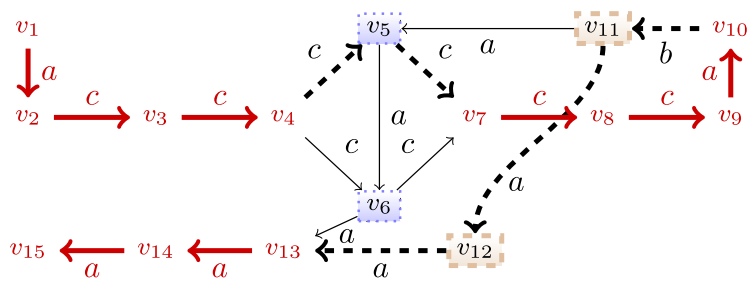

$$
\begin{array}{ll}
v_{j} \underset{b}{\stackrel{b}{\longrightarrow}} v_{i} & : \text { subpath in summary } \\
v_{j}=-\rightarrow & : \text { subpath outside summary } \\
v_{i} & : \text { vertex in } \text { Set }_{1} \\
v_{i} & : \text { vertex in } \text { Set }_{2}
\end{array}
$$

(b) A graph. A shortest simple $L$-labeled path $p_{1}$ (thick) from $v_{1}$ to $v_{15}$ is illustrated with thick edges. Dashed edges are eliminated in its summary (see Example 2).

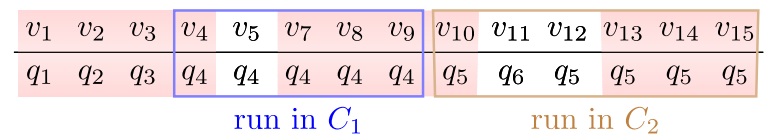

(c) Run of $A$ over $p_{1}$.

Fig. 2. Components, summaries and safe completions.

We also observe that in a summary, all cut symbols are clearly distinct by definition of strongly connected components. A summary therefore contains at most $M(M+2)=O\left(M^{2}\right)$ elements (vertices, labels and cut symbols), which is constant if $L$ is fixed. As a consequence, each summary can be represented with a logarithmic number of bits.

We next define a candidate summary as an alternative sequence of vertices and symbols or cut symbols of the form above.

Definition 5 (Candidate summary). We define as a candidate summary $S$ any sequence of vertices and labels of the form above; $S=\left(v_{1}, \alpha_{1}, \ldots, \alpha_{m}, v_{m+1}\right)$ where $\alpha_{i} \in \Sigma \cup$ Cuts for every $i \in[m]$, all cut symbols are distinct, and $m \leq M$. Similarly to Definition 3, we denote by $\mathrm{cut}_{C_{1}}, \ldots$, cut $_{C_{l}}$ the sequence of cut symbols appearing in $S$. Furthermore we define, for each $i \in[l]$, left $_{i}$ (resp. right $t_{i}$ ) as the vertex at left (resp. right) of cut $_{C_{i}}$ in $p$.

Every summary is clearly a candidate summary but a candidate summary needs not be the summary of any $L$-labeled path, let alone a simple one.

Definition 6. A path $p$ obtained by replacing each subsequence $\left(\right.$ left $_{i}$, cut $_{C_{i}}$, right $\left.t_{i}\right)$ with a simple $\Sigma_{C_{i}}^{*}$-labeled path from left to right $_{i}$ is called a completion of the candidate summary $S$.

By definition, completion and summary are inverse operations in the following sense:

Lemma 13. Let $S$ be the summary of an L-labeled path $p$ and let $p^{\prime}$ be a completion of S. Then, $p^{\prime}$ is an L-labeled path with summary S. 
For each candidate summary $S$ that happens to be a summary, we could compute in NL a completion of $S$. For instance, this can be done by repeatedly using a reachability oracle. By definition, this completion $p$ is an $L$-labeled path from a summary $S$. However, the path $p$ is not necessarily simple, even if $S$ is the summary of a simple path. The reason is that the paths $\left(p_{i}\right)_{i \in[l]}$ we have built between each left $t_{i}$ and right $t_{i}$ are not necessarily disjoint.

\subsection{Safe completions}

The completion of a summary needs not be a simple path. We therefore define in this section safe completions such that safe completions are always simple $L$-labeled paths, and reciprocally every shortest simple $L$-labeled paths is a safe completion of its summary.

For that purpose, we will define local domains $\operatorname{Set}_{1}, \ldots$, Set $t_{l}$ which are disjoint sets of vertices from $G$. For each $i \in[l]$, the safe completions require the path $p_{i}$ between left $_{i}$ and right $t_{i}$ to be $S_{e} t_{i}$-restricted. Consequently, these paths will be disjoint. To guarantee that reciprocally we can find a safe completion for the summary of a shortest simple $L$-labeled path, we define $S_{i} t_{i}$ to the set of vertices that might occur on a shortest $\Sigma_{C_{i}}^{*}$-labeled path from left $t_{i}$ to right $t_{i}$ that avoids all Set ${ }_{j}$ $(j<i)$ :

Definition 7 (Local domains). Let $S$ be a candidate summary. We denote by $\left(C_{i}, \text { left }_{i} \text {, right }\right)_{i \in[l]}$ its components as stated in Definition 5, and denote by $V(S)$ the set of vertices appearing in $S$. We define the local domains Set $_{i}$ recursively for each $i$ from 1 to $l$. The set $S_{e} t_{i}$ is defined as a subset of $V_{i}=V \backslash\left(V(S) \cup \cup_{j<i}\right.$ Set $\left._{j}\right)$, as follows. If there is no $V_{i}$-restricted $\Sigma_{C_{i}}^{*}$-labeled simple path $p$ from left $_{i}$ to right $_{i}$, then $S_{e} t_{i}=\emptyset$. Otherwise, we denote by $k_{i}$ the length of the shortest such path and define $S_{e} t_{i}$ as the set of vertices $y$ in $V_{i}$ that can be reached from left $t_{i}$ by a $V_{i}$-restricted $\Sigma_{C_{i}}^{*}$-labeled path $p$ of length at most $k_{i}-1$.

The following lemma is a direct consequence of Definition 7.

Lemma 14. Let $S$ be a candidate summary. Then all sets $V(S),\left(\operatorname{Set}_{i}\right)_{i \in[l]}$ from Definition 7 are disjoint.

Definition 8 (Safe completion). Let $p$ be a path with label in $L$ and summary $S$. We qualify $p$ as safe completion of summary $S$ if the following two conditions are satisfied: (a) all vertices appearing in $S$ are distinct and (b) for every $i \in[l]$, the path $p\left[\right.$ left $_{i}$, right $\left._{i}\right]$ is simple and Set $_{i}$-restricted.

By the definition and Lemma 14 , a safe completion is necessarily simple.

Lemma 15. Let $S$ be a candidate summary. Every safe completion of $S$ is a simple L-labeled path.

Example 3. The path $p_{1}$ defined in Example 2 is a safe completion, since $\operatorname{Set}_{1}=\left\{v_{5}, v_{6}\right\}$ and $\operatorname{Set}_{2}=\left\{v_{11}, v_{12}\right\}$, as illustrated in Fig. 2b. The definition of safe completions guarantees the paths replacing cut $_{C_{1}}$ and cut $_{C_{2}}$ are disjoint. Indeed we can check that $\left\{v_{5}\right\} \cap\left\{v_{11}, v_{12}\right\}=\emptyset$.

Being a safe completion is clearly more restrictive than being a simple path. However, it turns out that shortest simple paths are safe completions, as shown below. That means that the existence of a simple path is equivalent to the existence of a safe completion.

Lemma 16. Let $(G, x, y)$ be a $R S P Q(L)$ instance. Every shortest simple L-labeled path from $x$ to $y$ is a safe completion of its summary.

Proof. Let $p=\left(v_{1}, a_{1}, \ldots, a_{m}, v_{m+1}\right)$ be a shortest simple $L$-labeled path from $x$ to $y$. Assume that $p$ is not a safe completion. That means there is some $i_{0}$ and vertex $v$ between left $_{i_{0}}$ and right $i_{i_{0}}$ such that $v \notin$ Set $_{i_{0}}$. Equivalently, if $p$ is not a safe completion, then there exists some $i$ and some vertex $v$ on the path that satisfy one of the following two properties $\mathcal{P}_{1}(i)$ or $\mathcal{P}_{2}(i):(1) v$ satisfies $\mathcal{P}_{1}(i)$ if there is $j>i$ such that $v$ is visited between left $_{j}$ and right ${ }_{j}$, and $v \in$ Set $_{i}(2) v$ satisfies $\mathcal{P}_{2}(i)$ if no vertex $v^{\prime}$ satisfies $P_{1}(i), v$ is visited between left $_{i}$ and right $t_{i}$, and $v \notin$ Set $_{i}$. Intuitively, case (2) covers the case where the path between left $t_{i}$ and right $t_{i}$ stays within $V_{i}$ but fails to stay within Set $_{i}$.

We choose a minimal such $i$. For each of the two cases, we will construct a path $p^{\prime}$ shorter than $p$ from $x$ to $y$. We then prove that $p^{\prime}$ is an $L$-labeled simple path, which contradicts our assumption that $p$ is the shortest such path.

Case (1): let $v$ be a vertex satisfying property $\mathcal{P}_{1}(i)$. Then, by definition of $\operatorname{Set}_{i}$, there is a Set $t_{i}$-restricted $\Sigma_{C_{i}}^{*}$-labeled simple path $s p$ from left $t_{i}$ to $v$ that is shorter than the subpath $p\left[\right.$ left $_{i}$, right $\left.t_{i}\right]$ and, consequently, shorter than $p\left[\right.$ left $\left._{i}, v\right]$. Let $p^{\prime}$ be the path obtained from $p$ by replacing $p\left[\right.$ left $\left._{i}, v\right]$ with $s p$. This path $p^{\prime}$ is shorter than $p$. For the remainder of the proof we assume that $v$ is the last visited vertex in $p$ satisfying property $\mathcal{P}_{1}(i)$ and we define $p^{\prime}$ accordingly.

Case (2): let $v^{\prime}$ be a vertex satisfying property $\mathcal{P}_{2}(i)$. There is a Set $_{i}$-restricted $L$-labeled simple path $s p$ between left and right $t_{i}$ that is shorter than $p\left[\right.$ left $_{i}$, right $t_{i}$. We choose $p^{\prime}$ as the path obtained from $p$ by replacing $p\left[\right.$ left $t_{i}$,right $\left.t_{i}\right]$ with $s p$. Furthermore, for homogeneity of the proof, we define $j=i$ and $v=\operatorname{right}_{i}\left(v\right.$ is not the vertex satisfying property $\left.\mathcal{P}_{2}(i)\right)$. 
The remainder of the proof is common to the two cases. We need to prove that $p^{\prime}$ is a simple $L$-labeled path. We first prove that $p^{\prime}$ is an $L$-labeled path. Let $\rho^{\prime}$ be the run of $L$ over $p^{\prime}$. Let $w$ be the word formed by the labels of the subpath

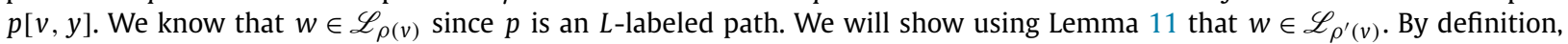
$\rho^{\prime}\left(\right.$ left $\left._{i}\right)$ belongs to the component $C_{i}$ and the path $s=p^{\prime}\left[\right.$ left $\left.t_{i}, v\right]$ is $\Sigma_{C_{i}}^{*}$-labeled, hence $\rho^{\prime}(v) \in C_{i}$ by Lemma 9. Furthermore $\rho(v) \in C_{j}$ and $C_{j}$ is reachable from $C_{i}$. In addition, by definition of a summary, there are at least $M+1$ vertices $v^{\prime \prime}$ of $p$ after $v$ (including $v$ ) such that $\rho\left(v^{\prime \prime}\right) \in C_{j}$, and therefore the $M$ labels following vertex $v$ in $p$ belong to $\Sigma_{C_{j}}$ by Lemma 9 again. The definition of components $C_{i}, C_{j}$ guarantees that $q_{1}, q_{2}$ admit loops. We have thus proved that $q_{1}=\rho^{\prime}(v)$ and

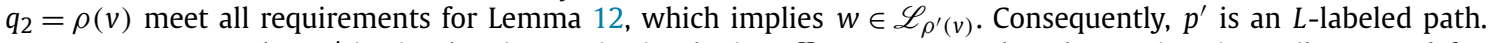

We now prove that $p^{\prime}$ is simple. Since $p$ is simple, it suffices to prove that the vertices in $s p$ (between left $t_{i}$ and $v$ ) are disjoint with other vertices in $p^{\prime}$. Notice that all intermediate vertices of $s p$ belong to Set $t_{i}$. By minimality of $i$, for all $i^{\prime}<i$, the vertices between left $_{i^{\prime}}$ and right $i_{i^{\prime}}$ belong to Set $_{i^{\prime}}$ and, since Set $i_{i^{\prime}}$ and Set $_{i}$ are disjoint (Lemma 14), do not belong to Set ${ }_{i}$. Consequently, there is no vertex $v^{\prime}$ before left $_{i}$ such that $v^{\prime}$ belong to Set $t_{i}$. By construction, in the two cases (1) and (2), there is no vertex $v^{\prime}$ after $v$ such that $v^{\prime}$ belongs to Set ${ }_{i}$. This concludes the proof.

We observe that many of our summary and completion definitions were actually tailored for this Lemma 16: our proof by contradiction explains for instance why our definition of Set $t_{i}$ guarantees the existence of a safe completion. And our application of Lemma 12 in that proof explains why our summaries feature $M+2$ vertices of each component.

We next show how a summary admitting a safe completion can be completed in logarithmic space into a simple path.

\subsection{An algorithm for RSPQ via safe completions}

We next introduce Algorithm 1, show that it computes simple L-labeled path from $x$ to $y$, and analyze its complexity.

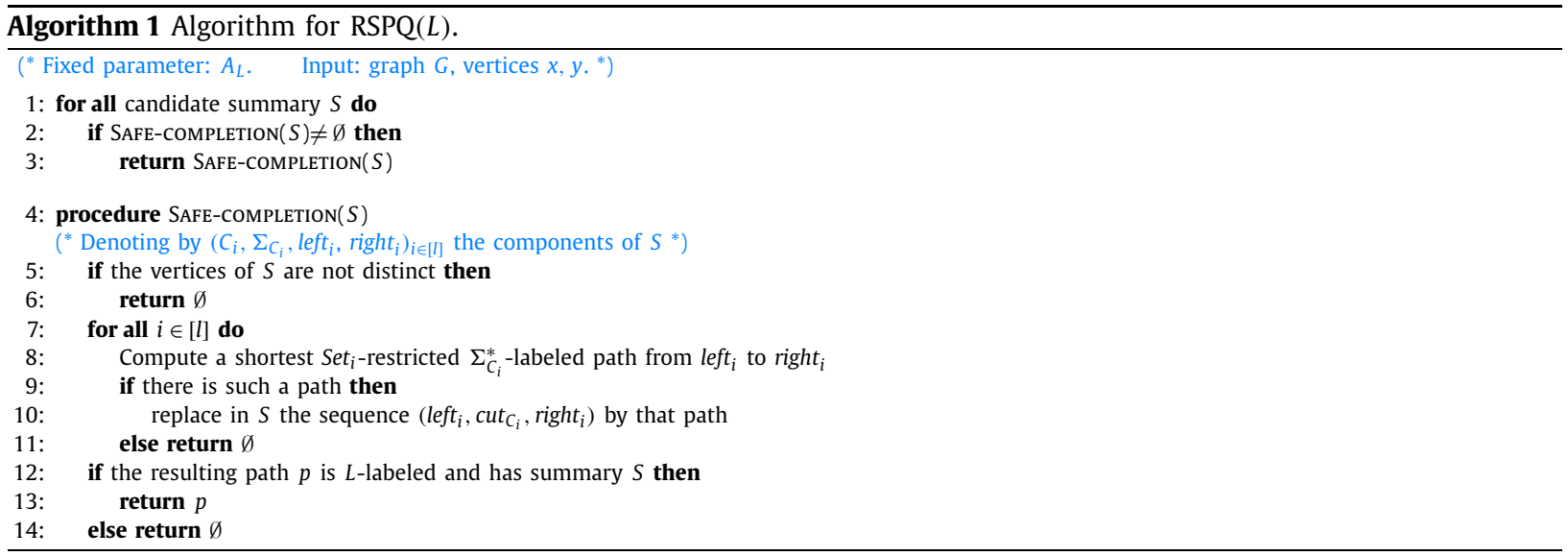

Theorem 1. Algorithm 1 returns a simple L-labeled path from $x$ to $y$ if there is one. The algorithm can be implemented in NL.

We first prove that local domains Set $_{i}$ can be computed in logarithmic space.

Lemma 17. Let $L$ be a fixed language in $\mathcal{C}_{\text {tract. }}$ The following problem $P_{\text {Set }}$ is in $\mathrm{NL}$. Given an instance $(G, x, y)$ of $R S P Q(L)$, a candidate summary $S$, a vertex $z$ and an integer $i$, decide whether $z \in$ Set $_{i}$.

Proof. For each $k \geq 0$, let $P_{\text {Set }}^{k}$ be the decision problem $P_{\text {Set }}$ with the restriction $i \leq k$ : $(G, x, y, S, z, i) \in P_{\text {Set }}^{k}$ iff $(G, x, y, S, z, i) \in P_{\text {Set }}$ and $i \leq k$.

Clearly, the number $l$ of cuts in a summary $S$ as in Definition 4, is bounded by the number $K$ of strongly connected components of $L$. Consequently, $P_{S e t}=P_{S e t}^{K}$. Notice that $K$ is a constant that does not depend on the instance. Let us prove, that $P_{\text {Set }}^{k} \in \mathrm{NL}$ for each $k \in[0, K]$. The proof is by induction on $k$. If $k=0, P_{\text {Set }}^{0}$ always returns False because Set $i$ is not defined for $i=0$. So $P_{\text {Set }}^{0}$ is trivially in NL. Assume, by induction, that $P_{\text {Set }}^{k} \in \mathrm{NL}$. It suffices to show that there is an NLalgorithm for $P_{\text {Set }}^{k+1}$ using $P_{\text {Set }}^{k}$ as oracle. Since $\mathrm{NL}^{\mathrm{NL}}=\mathrm{NL}$ (Lemma 2), this implies that $P_{\text {Set }}^{k+1} \in$ NL. Let $(G, x, y, S, z, i)$ be an instance of $P_{\text {Set }}^{k+1}$. If $i \leq k$, we return the same answer as the oracle $P_{\text {Set }}^{k}$. If $i=k+1$, using the definition and notations of $\operatorname{Set}_{k+1}$, the problem essentially boils down to computing the distances between the vertices left $t_{k+1}$ and $z$ on one hand, left $t_{k+1}$ and right $_{k+1}$ on the other hand in the graph $G^{\prime}=\left(V^{\prime}, E^{\prime}\right)$ where $V^{\prime}=V_{k+1} \cup\left\{\right.$ left $_{k+1}$, right rit $_{k+1}$ and $E^{\prime}$ is the set of $\Sigma_{C_{k+1}}$-labeled edges of $G\left[V^{\prime}\right]$. In order to remain within logarithmic space, the graph $G^{\prime}$ is not stored in memory but simulated. The distance computation can thus clearly be performed in non deterministic log-space using the oracle $P_{\text {Set }}^{k}$. 
Lemma 18. Let $L$ be a fixed language in $\mathcal{C}_{\text {tract }},(G, x, y)$ an instance of $R S P Q(L)$ and $S$ a summary. Procedure SAFE-Completion $(S)$ from Algorithm 1 returns in $\mathrm{NL}$ a safe completion $p$ from $x$ to $y$ if there is any, and returns $\emptyset$ otherwise.

Proof. The correctness of the procedure is immediate as it matches the definition of safe completions. We still have to check the complexity. To achieve logarithmic space, we do not store the $S_{e} t_{i}$ in memory: we only need to check on-the-fly if a given vertex belongs those sets, using Lemma 17 . This proves the algorithm can easily be implemented in NL.

We can now prove Theorem 1. By Lemmas 15 and 16, there exists a safe completion from $x$ to $y$ if and only if there is a simple path from $x$ to $y$. Lemma 16 implies that Algorithm 1 identifies a safe completion if there is one, so the algorithm is correct. The complexity is in NL as candidate summaries have constant size and therefore can be enumerated in NL, whereas completions are computed in NL according to Lemma 16. This concludes the proof of Theorem 1.

Notice that we can easily adapt Algorithm 1 so that it outputs a shortest path for positive instances. The main theorem summarizes our results, combining Lemma 4 with Theorem 1.

Theorem 2. Let $L$ be a regular language. Then, $R S P Q(L)$ is in $\mathrm{NL}$ if $L \in \mathcal{C}_{\text {tract }}$ and is NP-complete otherwise.

\subsection{Towards a complete classification}

We have partitioned $\mathrm{RSPQ}(L)$ problems into NL and NP-complete problems. We next refine the classification within the class of NL problems.

Lemma 19. For every regular language $L, R S P Q(L) \in \mathrm{AC}^{0}$ if $L$ is finite, otherwise $R S P Q(L)$ is NL-hard.

The proof is based on a reduction from the following NL-complete problem [24].

\section{Reachability}

Input: A directed graph $G$ and two vertices $x, y$ in $G$

Question: Is there a path from $x$ to $y$ ?

Proof. (Membership) For a finite language $L, \operatorname{RSPQ}(L)$ can easily be defined by a first-order formula, more precisely a disjunction of conjunctive formulas. Consequently, $\operatorname{RSPQ}(L) \in \mathrm{AC}^{0}[12]$.

(Hardness) We exhibit a reduction from Reachability. Let $L$ be an infinite regular language. By the Pumping Lemma, there exist non empty words $u, v, w$ such that $u v^{*} w \subseteq L$. We build a db-graph $G^{\prime}$ from $G$ by first labeling every edge of $G$ with $v$, and then adding two vertices $x^{\prime}$ and $y^{\prime}$ with edges $\left(x^{\prime}, u, x\right)$ and $\left(y, w, y^{\prime}\right)$. There is a (not necessarily simple) path from $x$ to $y$ in $G$ iff there is an $L$-labeled simple path from $x^{\prime}$ to $y^{\prime}$ in $G^{\prime}$. Consequently, $\operatorname{RSPQ}(L)$ is NL-hard.

Our results so far can be summarized in the following trichotomy which refines Theorem 2 .

Theorem 3. Let $L$ be a regular language. The complexity of $R S P Q(L)$ can be determined as follows.

1. L is finite: $R S P Q(L) \in \mathrm{AC}^{0}$;

2. $L \in \mathcal{C}_{\text {tract }}$ and $L$ is infinite: $R S P Q(L)$ is NL-complete;

3. $L \notin \mathcal{C}_{\text {tract }}: R S P Q(L)$ is NP-complete.

\section{Variations}

In this Section, we analyze the computational complexity of variations of the main problem: allowing $\epsilon$-edges, finding shortest paths in weighted graphs and finding paths that minimize repetitions of vertices.

\subsection{Db-graphs with $\epsilon$-edges}

We observed in Lemma 4 that $\operatorname{RSPQ}(L)$ does not become harder if we allow edges labeled by non empty words. However, we have not discussed yet the complexity of $\operatorname{RSPQ}(L)$ when we allow edges labeled by $\epsilon$. We name $\operatorname{RSPQ}^{\epsilon}(L)$ this variation of $\operatorname{RSPQ}(L)$. We show that the class of tractable languages $\operatorname{RSPQ}^{\epsilon}(L)$ corresponds to the languages closed under subwords, and consequently is a strict subset of $\mathcal{C}_{\text {tract }}$.

Theorem 4. Let $L$ be a regular language. The complexity of $R S P Q^{\epsilon}(L)$ can be determined as follows.

1. L is empty: $R S P Q^{\epsilon}(L)$ is trivial i.e. does not contain positive instances; 
2. L is non empty and closed under subwords: $R S P Q^{\epsilon}(L)$ is NL-complete;

3. $L$ is not closed under subwords: $R S P Q^{\epsilon}(L)$ is NP-complete.

Proof. 1) is straightforward.

2) The membership in NL is straightforward: for any subword closed language $L$, there exists a simple $L$-labeled path from $x$ to $y$ in $G$ if and only if there exists an $L$-labeled path (simple or not) from $x$ to $y$ in $G$. This can obviously be checked in NL. We next prove that $\operatorname{RSPQ}^{\epsilon}(L)$ is NL-hard. Since $L$ is not empty, let $w$ be a word in $L$. The reduction is identical to the reduction of Lemma 19. We choose $u=v=\epsilon$ and $w$ as defined above.

3) Since $L$ is not closed under subwords, there exist words $u, v, w$ such that $u v w \in L$ and $u w \notin L$. The reduction is identical to the reduction of Lemma 4. We choose $w_{1}=w_{2}=\epsilon, w_{l}=u, w_{m}=v$ and $w_{r}=w$.

\subsection{Shortest path in weighted graphs}

In this section, we consider the following problem:

\section{weighted-RPQ $(L, C)$}

Input: A db-graph $G$, two vertices $x$ and $y$ and a positive weight function $W$ over the edges of $G$

Objective: Find a simple path $L$-labeled path $p$ from $x$ to $y$ with minimal weight $W(p)$ if such a path exists

The problem can be solved with a straightforward generalization of Algorithm 1 . Edges of weight 0 are the only ones that require special care. Indeed, in the proof of Lemma 16, we start from a shortest path $p$ and build a path $p^{\prime}$ shorter than $p$ with the goal of showing a contradiction. When both $p$ and $p^{\prime}$ can have weight 0 , there may be no contradiction. To overcome this problem, one can for instance define weights over a product order to count the number of edges with weight 0 : if the weights are originally defined over some ordered set $W$, we assign to each edge $e$ the weight $(w, 0)$ if $e$ has weight $w>0$, and $(w, 1)$ otherwise. The weights of the edges along a path are summed componentwise, and paths are compared based on a lexicographic order. This means that the number of edges with weight 0 along a path is solely used to break ties.

\subsection{Paths that minimize repetitions of vertices}

We next show that our technique and results extend to generalizations of the simple path problem that do not forbid but only limit repetitions of vertices along the path.

Definition 9. Let $p$ be a path and $v_{1}, \ldots, v_{m}$ be the sequence of vertices appearing in $p$. We define rep $(p)$ as the multiset keeping only vertices $v_{i}$ for which there is $v_{j}, j<i$ such that $v_{i}=v_{j}$. We denote by $n b r e p(p)$ the cardinality of $\operatorname{rep}(p)$.

Clearly $p$ is a simple path if and only if $\operatorname{nbrep}(p)=0$. We consider the following problem:

$\operatorname{rep}-\operatorname{RPQ}(L)$

Input: $\mathrm{A} d \mathrm{db}$-graph $G$, two vertices $x$ and $y$ and an integer $k \geq 0$

Question: Is there an $L$-labeled path $p$ from $x$ to $y$ such that $n b r e p(p) \leq k$ ?

Lemma 20. Let $L \in \mathcal{C}_{\text {tract. }}$ Then there is a constant $K_{L}$ such that for every db-graph $G$ and L-labeled path $p$ from $x$ to $y$ in $G$, there exists an L-labeled path $p^{\prime}$ from $x$ to $y$ with $\operatorname{nbrep}\left(p^{\prime}\right) \leq K_{L}$.

Proof. Let $p$ be an $L$-labeled path from $x$ to $y$ that minimizes $n b r e p(p)$. We will prove that all occurrences of a given vertex $v$ appear in the summary $S$ except at most one. This is sufficient for the proof since the size of the summary $S$ is bounded by a constant. Assume, for the sake of contradiction, that $p$ is of the form $\left(v_{1}, a_{1}, \ldots a_{l}, v_{l+1}\right)$ and contains two occurrences $v_{i}, v_{j}$ of the same vertex such that $v_{i}$ and $v_{j}$ are not in the summary of $p$. Let $p^{\prime}=\left(v_{1}, \ldots v_{i}, a_{j}, v_{j+1}, \ldots, v_{l+1}\right)$. Then, $\operatorname{nbrep}\left(p^{\prime}\right)<\operatorname{nbrep}(p)$, and $p^{\prime}$ is an $L$-labeled path by Lemma 11 .

Theorem 5. Let $L \in \mathcal{C}_{\text {tract. }}$ Then, rep-RPQ $(L) \in \mathrm{NL}$.

Proof. Given a db-graph $G$ and a multiset $A$ of vertices in $G$, we define the graph $c(G, A)$ as follows. We start from $G$ and for each vertex $x$ in $A$ we add a new vertex in $G$ which is a copy of $x$ i.e. has the same incoming and outgoing edges as $x$ has.

By construction, we have the following property: there exists an $L$-labeled simple path from $x$ to $y$ in $c(G, A)$ iff there exists an $L$-labeled path $p$ from $x$ to $y$ in $G$ such that $r e p(p) \subseteq A$. Consequently, it suffices to enumerate all multisets $A$ of 
Table 1

A family of regular expressions for $\mathcal{C}_{\text {tract }}$

$$
\begin{aligned}
\Psi_{\text {seq }}::= & w+\epsilon\left|A^{\geq k}+\epsilon\right| \Psi_{\text {seq }} \Psi_{\text {seq }} \\
\Psi_{\text {tr }}::= & w \Psi_{\text {seq }} w^{\prime} \mid \Psi_{\text {tr }}+\Psi_{\text {tr }} \\
& \text { where } w, w^{\prime} \in \Sigma^{*}, A \subseteq \Sigma
\end{aligned}
$$

at most $k$ vertices and check if, for one of them, $c(G, A), x, y$ is a positive instance of RSPQ $(L)$. Thanks to Lemma 20 , we do not have to consider multisets of more than $K_{L}$ vertices.

Moreover, it is easily seen that $c$ is a function in FL. Thus, we obtain the following algorithm for rep-RPQ $(L)$.

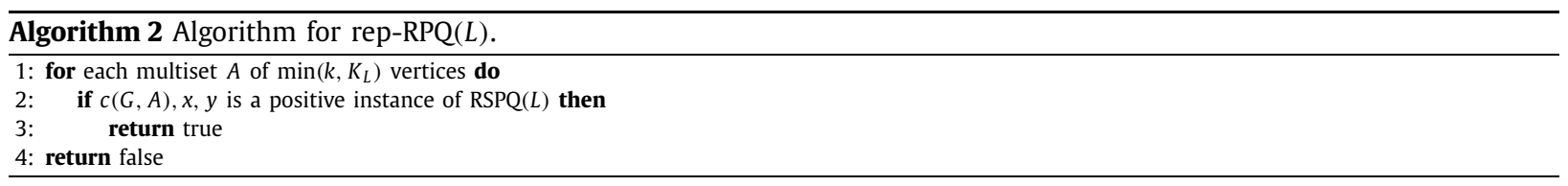

Other constraints on repetitions may be worth mentioning. It is obvious that Lemma 20 and therefore also Theorem 5 still hold if we define $n b r e p(p)$ as the maximal number of occurrences of a vertex in $p$. Or if we define $n b r e p(p)$ as the number of distinct vertices that are repeated (one or several times) in $p$.

\section{Characterization by regular expressions}

In this section, we propose two characterizations of $\mathcal{C}_{\text {tract }}$ languages. The first in terms of regular expressions and the second in terms of a pumping property. Unlike the other properties discussed before on the minimal DFA of $L$, the pumping property is expressed directly on the language $L$. The languages in $\mathcal{C}_{\text {tract }}$ are exactly those that can be expressed with an expression in the fragment $\Psi_{t r}$ defined in Table 1 . This fragment enforces restrictions on the concatenation of subexpressions: roughly speaking, only expressions of the form $e+\epsilon$ can be concatenated.

Example 4. For instance, the expression $a^{*}\left(b^{\geq 2}+\epsilon\right) c^{*}$ investigated in Example 1 belongs to the fragment $\Psi_{t r}$ (using notation $\left.c^{*}=c^{\geq 0}\right)$. Expression $a^{*} b a^{*}+(a+b)^{*}$, on the opposite, does not, but is clearly equivalent to $(a+b)^{\geq 0}$, which does. The following theorem, however, implies that $a^{*} b a^{*}$ is not equivalent to any expression from $\Psi_{t r}$.

Theorem 6. Let $L$ be a regular language. The three following statements are equivalent:

1. A language $L$ belongs to $\mathcal{C}_{\text {tract }}$.

2. $L$ is recognized by a regular expression in $\Psi_{t r}$.

3. There is an integer $i \geq 0$ such that for all words $w_{l}, w_{m}, w_{r} \in \Sigma^{*}$ and all non empty words $w_{1}, w_{2} \in \Sigma^{+}, w_{l} w_{1}^{i} w_{m} w_{2}^{i} w_{r} \in L$ implies $w_{l} w_{1}^{i} w_{2}^{i} w_{r} \in L$.

Proof. $1 \Rightarrow 2$ ) We next outline an algorithm to build the regular expression $e$ from $A_{L}$. Let $C_{1}, \ldots, C_{l}$ be the strongly connected components of $L$ in some topological order. For every $k \in\{0, \ldots, l\}$ and every sequence $1 \leq j_{1}<\cdots<j_{k} \leq l$, we denote by $L\left[j_{1}, \ldots, j_{k}\right]$ the set of all words from $L$ that stay for at least $2 M$ steps in each component $C_{j_{1}}, \ldots, C_{j_{k}}$, and stay for at most $2 M-1$ steps after entering in each other component.

Clearly, $L$ is the union of all $L\left[j_{1}, \ldots, j_{k}\right]$ over all sequences $j_{1}, \ldots, j_{k}$. We next show how to build an expression for $L\left[j_{1}, \ldots, j_{k}\right]$. We denote by $S_{1}, \ldots, S_{k}$ the components $C_{j_{1}}, \ldots, C_{j_{k}}$ and by $\Sigma_{1}, \ldots, \Sigma_{k}$ their alphabet. For any component $S_{i}$ and state $q$ in $S_{i}$, we can easily build an expression $H_{q}$ with language: $L\left(H_{q}\right)=\left\{w \in\left(\Sigma_{i}\right)^{M} \mid \exists q_{0} \in S_{i}, \Delta_{L}\left(q_{0}, w\right)=q\right\}$. The rationale for this definition is that words of $\left(\Sigma_{i}\right)^{M}$ are synchronizing within $S_{i}$ according to Lemma 12: for all $q, q_{1} \in S_{i}$ and $w \in L\left(H_{q}\right)$, we have $\Delta_{L}\left(q_{1}, w\right)=q$.

Let $i<k$ and $q \in S_{i}$. We build an expression $W_{q}$ for the set of all words $w$ that lead from $q$ to some state of $S_{i+1}$ while respecting the sequence of components. In other words, a word $w=a_{1} \ldots a_{m}$ belongs to $L\left(W_{q}\right)$ iff when we denote by $q_{j}$ the state $\Delta_{L}\left(q, a_{1} \ldots a_{j}\right)$, the sequence $q_{1} \ldots q_{m}$ satisfies the following properties ${ }^{2}$ :

- $q_{1} \notin S_{i}$

- $q_{m}$ is the first state of the sequence that belongs to $S_{i+1}$

\footnotetext{
${ }^{2}$ We require somewhat arbitrarily that the first letter of $w$ lets quit $S_{i}$, while the last letter of $w$ lets enter $S_{i+1}$ (i.e., is not in $\Sigma_{i+1}$ ).
} 
- there are at most $2 M$ states $q_{j}$ in the same component of $A_{L}$.

Similarly, for $i=k$, we build for any state $q \in S_{k}$ an expression $W_{q}$ for the set of all words $w$ that lead from $q$ to some final state while respecting the sequence of components, i.e., satisfying conditions similar to the above ones except that $q_{m}$ belongs to $F_{L}$ instead of $S_{i+1} . L\left(W_{q}\right)$ is a finite set of words having length at most $2 M^{2}$.

If $i_{L}$ belongs to $S_{1}$, we define the expression $e_{\text {init }}$ as $\epsilon$, otherwise $e_{\text {init }}$ is the set of all words that lead from $i_{L}$ to some state in $S_{1}$ while respecting the sequence of components. Rephrased differently, a word $w=a_{1} \ldots a_{m}$ belongs to $L\left(e_{\text {init }}\right)$ iff, when we denote by $q_{j}$ the state $\Delta_{L}\left(i_{L}, a_{1} \ldots a_{j}\right)$, the sequence $q_{1} \ldots q_{m}$ satisfies the following properties:

- $q_{m}$ is the first state of the sequence that belongs to $S_{1}$,

- there are at most $2 M$ states $q_{j}$ in the same component of $A_{L}$.

$e_{\text {init }}$ is a finite set of words having length at most $2 M^{2}$.

Claim 1: The expression $e_{0}^{\prime}$ defined by the following equations represents the language $L\left[j_{1}, \ldots, j_{k}\right]$

$$
\begin{aligned}
& e_{k}^{\prime}=\left(\Sigma_{k}\right)^{\geq M} \cdot\left(\bigcup_{q \in S_{k}} H_{q} \cdot W_{q}\right) \\
& e_{i}^{\prime}=\left(\Sigma_{i}\right)^{\geq M} \cdot\left(\bigcup_{q \in S_{i}} H_{q} \cdot W_{q}\right) \cdot e_{i+1}^{\prime} \text { for all } 1 \leq i<k \\
& e_{0}^{\prime}=e_{\text {init }} \cdot\left(\Sigma_{1}\right)^{\geq M} \cdot\left(\bigcup_{q \in S_{1}} H_{q} \cdot W_{q}\right) \cdot e_{1}^{\prime}
\end{aligned}
$$

The language of $e_{0}^{\prime}$ clearly contains $L\left[j_{1}, \ldots, j_{k}\right]$. The converse inclusion follows from Lemma 12 , which concludes the proof of Claim 1. We now define the expressions $e_{0}, \ldots, e_{k}$ recursively as follows (with $i$ ranging from 1 to $k-1$ included, in the second equation):

$$
\begin{aligned}
& e_{k}=\left(\left(\Sigma_{k}\right)^{\geq M}+\epsilon\right) \cdot\left(\bigcup_{q \in S_{k}} H_{q} \cdot W_{q}\right) \\
& e_{i}=\left(\left(\Sigma_{i}\right)^{\geq M}+\epsilon\right) \cdot\left(\bigcup_{q \in S_{i}} H_{q} \cdot W_{q}+\epsilon\right) \cdot e_{i+1} \\
& e_{0}=e_{\text {init }} \cdot\left(\left(\Sigma_{1}\right)^{\geq M}+\epsilon\right) \cdot\left(\bigcup_{q \in S_{1}} H_{q} \cdot W_{q}+\epsilon\right) \cdot e_{1}
\end{aligned}
$$

Claim 2: The language of $e_{0}$ contains $L\left[j_{1}, \ldots, j_{k}\right]$ and is contained in $L$.

The language of $e_{0}$ clearly contains the language of $e_{0}^{\prime}$, hence $L\left[j_{1}, \ldots, j_{k}\right]$ by Claim 1 . Let $w \in L\left(e_{0}\right)$. There exist $u_{0}, v_{0}, u_{1}, v_{1} \ldots, u_{n}$, and $v_{n}$ such that

- $w=u_{0} v_{0} u_{1} v_{1} \ldots u_{n} v_{n}$

- $u_{0} \in L\left(e_{\text {init }} \cdot\left(\left(\Sigma_{1}\right)^{\geq M}+\epsilon\right)\right)$

- $v_{n} \in L\left(\bigcup_{q \in S_{k}} H_{q} \cdot W_{q}\right)$

- for each $0 \leq i \leq n-1, v_{i} \in L\left(\bigcup_{q \in S_{i}} H_{q} \cdot W_{q}+\epsilon\right)$

- for each $1 \leq i \leq n, u_{i} \in L\left(\left(\Sigma_{i}\right)^{\geq M}+\epsilon\right)$.

Let $w^{\prime}$ be the word obtained from $w$ by replacing every $v_{i}$ equal to $\epsilon$ with an arbitrary word from $L\left(\bigcup_{q \in S_{i}} H_{q} \cdot W_{q}\right)$, and every $e_{i}^{\prime}$ equal to $\epsilon$ with an arbitrary word from $L\left(\left(\Sigma_{i}\right)^{\geq M}\right)$. Then $w^{\prime}$ belongs to $L\left(e_{0}^{\prime}\right)$ and in particular to $L$. Consequently, $w$ also belongs to $L$ by repeated applications of Lemma 12 . As $L\left(W_{q}\right)$ and $L\left(H_{q}\right)$ are finite sets of words for every state $q$, $e_{0}$ belongs to the fragment, which concludes the proof of Claim 2 .

$2 \Rightarrow 3$ ) It is easily seen that languages that satisfy Statement (3) are closed by union. Consequently, we consider a regular expression $\varphi \in \Psi_{t r}$ of the form $\varphi_{1} \cdot \ldots \cdot \varphi_{l}$ where $\varphi_{1}$ and $\varphi_{l}$ are words and $\varphi_{i}$ is a $\Psi_{\text {seq }}$-term for every $i \in\{2, \ldots, l-1\}$. For each $i \in[l]$, we denote by $L_{i}$ the language recognized by $\varphi_{i}$. Let $M$ be the size of $\varphi$, defined as the number of states in its Glushkov automaton (i.e., the number of symbols in the expressions when terms of the form $A \geq k$ are expanded). Let $u, v, w, w_{1}, w_{2}$ be words with $w_{1}$ and $w_{2}$ non empty such that $u w_{1}^{M} v w_{2}^{M} w \in L$. We want to prove that $u w_{1}^{M} w_{2}^{M} w \in L$. Using the usual pumping argument for automata, applied to the Glushkov automaton of the expression, one shows easily that there is some term $\varphi_{i}$ of the form $A^{\geq n}+\epsilon$ such that $u w_{1}^{M} \in L_{1} \ldots L_{i}$ (and $w_{1}^{M} v w_{2}^{M} w \in A^{\geq n} \cdot L_{i+1} \ldots L_{l}$ ). Notice that $\varphi_{i}$ cannot be of the form $w+\epsilon$ because $w_{1}^{M}$ necessarily "ends" inside a Kleene-star subexpression by definition of $M$. Similarly, there is some term $\varphi_{j}, j \geq i$ of the form $B^{\geq m}+\epsilon$ such that $u w_{1}^{M} v w_{2}^{M} \in L_{1} \ldots L_{j}$ and $w_{2}^{M} w \in L_{j} \ldots L_{l}$. Thus, 
$u w_{1}^{M} w_{2}^{M} w \in L_{1} \ldots L_{i} \cdot L_{j} \ldots L_{l}$. Furthermore, $L_{1} \ldots L_{i} \cdot L_{j} \ldots L_{l} \subseteq L$ : if $i=j$, then $L_{i} L_{j} \subseteq L_{j}$ due to the form of $L_{i}$, and if $i<j$, then $\epsilon \in L_{i+1} L_{j-1}$ because every intermediate term can be skipped. As a consequence, $u w_{1}^{M} w_{2}^{M} w \in L$.

$3 \Rightarrow 1$ ) Assume that $L$ satisfies Statement (3). Let $i$ be as stated in (3). Let $q_{1}, q_{2} \in Q_{L}$ such that $\operatorname{Loop}\left(q_{1}\right) \neq \emptyset, \operatorname{Loop}\left(q_{2}\right) \neq \emptyset$ and $q_{2} \in \Delta_{L}\left(q_{1}, \Sigma^{*}\right)$. Let $w_{1}, w_{2}, w_{l}, w_{m}, w_{r} \in \Sigma^{*}$ such that $w_{1} \in \operatorname{Loop}\left(q_{1}\right), w_{2} \in \operatorname{Loop}\left(q_{2}\right), \Delta_{L}\left(i_{L}, w_{l}\right)=q_{1}, \Delta_{L}\left(q_{1}, w_{m}\right)=q_{2}$ and $w_{r} \in \mathscr{L}_{q_{2}}$. We need to prove that $w_{2}^{M} w_{r} \in \mathscr{L}_{q_{1}}$. Let $w=w_{l} w_{1}^{M} w_{m} w_{2}^{M} w_{r} \in L$. We consider two cases: $i \leq M$ and $i>M$. Case $i \leq M: w=w_{l} w_{1}^{M-i} w_{1}^{i} w_{m} w_{2}^{i} w_{2}^{M-i} w_{r}$. By hypothesis, we then have $w_{l} w_{1}^{M-i} w_{1}^{i} w_{2}^{i} w_{2}^{M-i} w_{r}=w_{l} w_{1}^{M} w_{2}^{M} w_{r} \in L$. Consequently, $w_{2}^{M} w_{r} \in \mathscr{L}_{q_{1}}$.

Case $i>M$ : By the classical pumping lemma, there exist integers $k, k^{\prime}>0$ such that for every $j, j^{\prime} \geq 0$, the word $w_{l} w_{1}^{M+k j} w_{m} w_{2}^{M+k^{\prime} j^{\prime}} w_{r}$ belongs to $L$. Consequently, for every $i_{1}, i_{2} \geq 0$, we can find a word $w^{\prime} \in w_{1}^{*} w_{m} w_{2}^{*}$ such that $w_{l} w_{1}^{i+i_{1}} w^{\prime} w_{2}^{i+i_{2}} w_{r} \in L$, which by hypothesis implies $w_{l} w_{1}^{i+i_{1}} w_{2}^{i+i_{2}} w_{r} \in L$. Observe that $\Delta\left(i_{L}, w_{l} w_{1}^{i+i_{1}}\right)=q_{1}$, so that $w_{2}^{i+i_{2}} w_{r} \in \mathscr{L}_{q_{1}}$ for every $i_{2} \geq 0$. By the usual pumping argument, $w_{2}^{M} w_{r} \in \mathscr{L}_{q_{1}}$.

The two characterizations of $\mathcal{C}_{\text {tract }}$ in Theorem 6 imply the following results.

Corollary 1. $\mathcal{C}_{\text {tract }}$ is closed under intersection, union and word reversal.

The closure under intersection and union of $\mathcal{C}_{\text {tract }}$ is a consequence of the second statement of Theorem 6 and the closure under word reversal is a consequence of the third statement of the same theorem.

An NFA $A$ is pseudo-acyclic if every loop in $A$ is a self-loop (i.e. a transition from a state to itself). Since in the third characterization of $\mathcal{C}_{\text {tract }}$ in Theorem 6 expressions under a Kleene star are unions of symbols, we obtain the following result.

Corollary 2. Let $L \in \mathcal{C}_{\text {tract }}$. Then, $A$ is recognizable by a pseudo-acyclic NFA.

The converse is not true since $a^{*} b a^{*}$ is not in $\mathcal{C}_{\text {tract }}$ but recognizable by a pseudo-acyclic NFA.

\section{Recognition of tractable languages}

The following theorem establishes the complexity of deciding if $\operatorname{RSPQ}(L)$ is tractable (i.e. deciding if $\operatorname{RSPQ}(L)$ can be computed in polynomial time). We consider different representations of $L$ (DFAs, NFAs and regular expressions).

Theorem 7. Testing whether a regular language $L$ belongs to $\mathcal{C}_{\text {tract }}$ is:

1. NL-complete if $L$ is given by a DFA;

2. PSPACE-complete if $L$ is given by an NFA (resp. a regular expression).

Before proving this theorem, we need some useful lemmas.

Lemma 21 (Folklore). There is an $\mathrm{L}$ transducer that, given two DFAs $A_{1}$ and $A_{2}$ that respectively recognize the languages $L_{1}$ and $L_{2}$, returns a DFA that recognizes the language $L_{1} \cap L_{2}$ (resp. $\left.L_{1} \cup L_{2}, L_{1} \backslash L_{2}\right)$.

Proof. The classical construction by product of the two DFAs can be done by an $\mathrm{L}$ transducer.

The next lemma permits to consider only minimal DFAs.

Lemma 22. Let $\mathcal{L}$ be a class of regular languages, such that we can test in NL whether a minimal DFA recognizes a language in $\mathcal{L}$.

1. testing whether a DFA recognizes a language in $\mathcal{L}$ is in $\mathrm{NL}$;

2. testing whether an NFA recognizes a language in $\mathcal{L}$ is in PSPACE.

Proof. 1) We will exhibit an NL transducer $t$ that given a DFA $A_{L}$ returns a minimal DFA $A_{L}^{\prime}$ equivalent to $A_{L}$. We construct $t$ as the composition of two transducers $t_{1}$ and $t_{2}$, where $t_{1}$ removes from $A_{L}$ all states non reachable from the initial state and $t_{2}$ merges all Nerode-equivalent states. This composition of two NL transducers is an NL transducer [24].

The log-space algorithm for $t_{1}$ uses an oracle for the problem Reachability and the log-space algorithm for $t_{2}$ uses an oracle for state equivalence. This problem of deciding given $q_{1}$ and $q_{2}$, whether $\mathscr{L}_{q_{1}}=\mathscr{L}_{q_{2}}$, is actually in NL since it can be reduced to Emptiness using L transducers of Lemma 21. 
2) We determinize the automaton and then apply an NL algorithm $\mathcal{A}$ to recognize $\mathcal{L}$ on the deterministic automaton thus obtained. To achieve the polynomial bound on space, the deterministic automaton is actually simulated on the fly instead of stored in memory.

Lemma 23. There is an $\mathrm{L}$ transducer that given a minimal DFA $A_{L}$ and a state $q$ of $A_{L}$ computes a DFA that recognizes the language $\operatorname{Loop}(q)^{M} \mathscr{L}_{q}$.

Proof. Let $L^{\prime}=\operatorname{Loop}(q)^{M} \mathscr{L}_{q}$. We construct a DFA $A_{L^{\prime}}=\left(Q_{L^{\prime}}, i_{L^{\prime}}, F_{L^{\prime}}, \Delta_{L^{\prime}}\right)$ as follows. This construction can clearly be computed by an $\mathrm{L}$ transducer.

- $Q_{L^{\prime}}=Q_{L} \times[0, M]$

- $i_{L^{\prime}}=(q, 0)$;

- $F_{L^{\prime}}=F_{L} \times\{M\}$;

- Let $\left(q_{1}, i\right) \in Q_{L^{\prime}}$ and $a \in \Sigma$. Then $\Delta_{L^{\prime}}\left(\left(q_{1}, i\right), a\right)=\left(\Delta_{L}\left(q_{1}, a\right), i+1\right)$ if $q_{1}=q$ and $i<M$. Otherwise, $\Delta_{L^{\prime}}\left(\left(q_{1}, i\right), a\right)=$ $\left(\Delta_{L}\left(q_{1}, a\right), i\right)$.

Let us prove that $A_{L^{\prime}}$ recognizes the language $L^{\prime}$. Let $w \in L^{\prime}=\operatorname{Loop}(q)^{M} \mathscr{L}_{q}$. We decompose $w$ as $w=w_{1} w_{2}$ where $w_{1} \in \operatorname{Loop}(q)^{M}$ and $w_{2}$ is accepted by $A_{L}$ starting from $q$. By construction $\left.\Delta_{L^{\prime}}(q, 0), w_{1}\right)=(q, M)$ and $w_{2}$ is accepted by $A_{L^{\prime}}$ starting from $(q, M)$. Thus, $w$ is accepted by $A_{L^{\prime}}$.

Let $w$ be a word accepted by $A_{L^{\prime}}$. By construction, we easily see that $w$ is accepted by $A_{L}$ starting from $q$. Additionally, the run of $w$ over $A_{L}$ contains at least $M+1$ occurrences of the state $q$. Consequently, $w=w_{1} w_{2}$ where $w_{1} \in \operatorname{Loop}(q)^{M}$ and $w_{2}$ is accepted by $A_{L}$ starting from $q$. Thus $w \in L^{\prime}$.

Lemma 24. We can decide in NL whether Loop $\left(q_{2}\right)^{\mathrm{M}} \mathscr{L}_{q_{2}} \subseteq \mathscr{L}_{q_{1}}$ given a minimal DFA together with two states $q_{1}$ and $q_{2}$.

Proof. Combining Lemmas 21 and 23, we obtain an L transducer that, given a minimal DFA $A_{L}$ and two states $q_{1}, q_{2}$, returns a DFA that recognizes the language $\operatorname{Loop}\left(q_{2}\right)^{M} \mathscr{L}_{q_{2}} \backslash \mathscr{L}_{q_{1}}$. We thus reduce our problem to the Emptiness problem that is in NL.

With the help of those lemmas we finally prove Theorem 7.

Proof. 1) Membership: let $A_{L}$ be a minimal DFA. The proof is based on the characterization of Lemma 6: for each pair of states $q_{1}, q_{2}$, we check in NL if they admit a loop and if $q_{2}$ is accessible from $q_{1}$. Then we check $\operatorname{Loop}\left(q_{2}\right)^{M} \mathscr{L}_{q_{2}} \subseteq \mathscr{L}_{q_{1}}$, still in NL according to Lemma 24. We thus check in NL whether $L \in \mathcal{C}_{\text {tract }}$ when $L$ is given by a minimal DFA which, together with Lemma 22, concludes our proof.

Hardness: we exhibit a reduction from Emptiness. Let $L \subseteq \Sigma^{*}$ and $L^{\prime}=1^{+} L 1^{+}$where $1 \notin \Sigma$. Moreover, we assume that $\epsilon \notin L$. Emptiness clearly remains NL-complete with this restriction. Our reduction maps a DFA $A_{L}$ that recognizes $L$ to a DFA $A_{L^{\prime}}$ that recognizes $L^{\prime}$. First, we prove that $L=\emptyset$ iff $L^{\prime} \in \mathcal{C}_{\text {tract }}$. If $L=\emptyset$ then $L^{\prime}=\emptyset \in \mathcal{C}_{\text {tract }}$. Conversely, assume that $L^{\prime} \in \mathcal{C}_{\text {tract }}$, and let $w \in \Sigma^{+}$. By construction, $1^{i} 1^{i} \notin L^{\prime}$ for every $i \geq 0$. As a consequence, Theorem 6 (using $w_{l}=w_{r}=\epsilon$ ) yields some $i \geq 0$ such that $1^{i} w 1^{i} \notin L^{\prime}$. Thus, $w \notin L$, hence $L$ is empty.

It remains to prove that the reduction can be computed with an $L$ transducer. We construct $A_{L^{\prime}}$ from $A_{L}$ as follows. We add a state $i_{L^{\prime}}$ that will be the initial state of $A_{L^{\prime}}$ and a state $f_{L^{\prime}}$ that will be the unique final state of $A_{L^{\prime}} . \Delta_{L^{\prime}}$ is the extension of $\Delta_{L}$ defined as follows:

- $\Delta_{L^{\prime}}\left(i_{L^{\prime}}, 1\right)=i_{L^{\prime}}$ and $\Delta_{L^{\prime}}\left(i_{L^{\prime}}, a\right)=i_{L}$ for every symbol $a \in \Sigma$.

- For every final state $q \in F_{L}, \Delta_{L^{\prime}}(q, 1)=f_{L^{\prime}}$, and $\Delta_{L^{\prime}}\left(f_{L^{\prime}}, 1\right)=f_{L^{\prime}}$.

2) The membership is a direct consequence of (1) and Lemma 22. To prove the hardness result we will exhibit a reduction from Universality. Consider the alphabet $\Sigma=\{0,1, a, b\}$ and two languages $L \subseteq\{0,1\}^{*}$ and $L^{\prime}=(0+1)^{*} a^{*} b a^{*}+L a^{*}$. Let us prove that $L=\{0,1\}^{*}$ iff $L^{\prime} \in \mathcal{C}_{\text {tract }}$. Assume that $L=\{0,1\}^{*}$. Then $L^{\prime}=(0+1)^{*} a^{*} b a^{*}+(0+1)^{*} a^{*}=(0+1)^{*} a^{*}(b+\epsilon) a^{*} \in \mathcal{C}_{\text {tract }}$ according to Theorem 6 . Conversely, assume $L^{\prime} \in \mathcal{C}_{\text {tract }}$. Let us prove that every word $w \in\{0,1\}^{*}$ belongs to $L$. By definition of $L^{\prime}, w a^{M} b a^{M} \in L^{\prime}$ for every $M \geq 0$. Consequently, $w a^{M} a^{M} \in L^{\prime}$ for some $M \geq 0$ since $L^{\prime} \in \mathcal{C}_{\text {tract }}$. Thus $w \in L$.

\section{Other minor results}

\subsection{Parametrized complexity}

The next section focuses on the parametrized complexity of the RSPQ problem. 


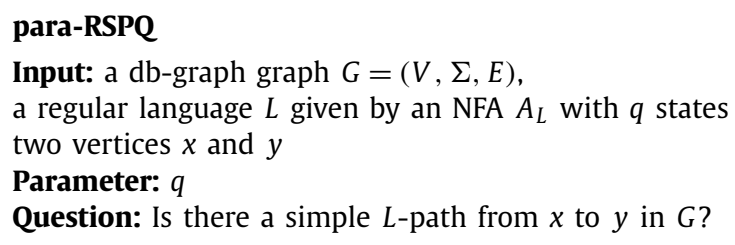

Our initial goal was to determine the parametrized complexity para-RSPQ $\left(\mathcal{C}_{\text {tract }}\right)$ when $L$ is restricted to $\mathcal{C}_{\text {tract }}$. Unfortunately, we could only partially reach this goal. We first address the parametrized complexity of RSPQs when the parameter is the size of the path.

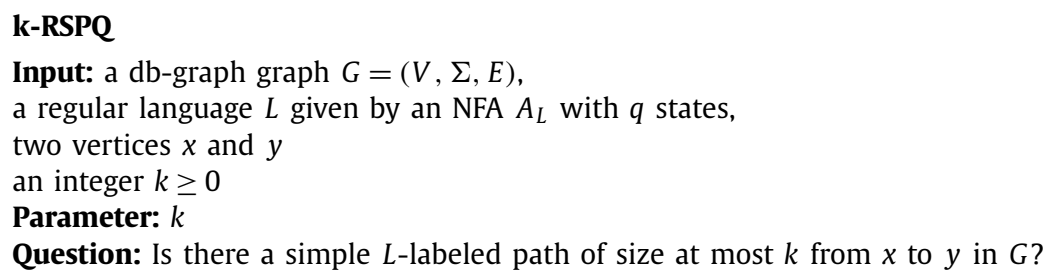

Theorem 8. $k$-RSPQ is FPT. More precisely, the problem is solvable in time $2^{O(k)} q \cdot|G| \cdot \log |G|$.

The proof is based on the Color Coding method [1]. Let $V$ be a finite set. A $k$-coloring of $V$ is a function $c: V \rightarrow[k]$. A set $S \subseteq V$ is colorful for $c$ if $c(x)=c(y) \Rightarrow x=y$ for every $x, y \in S$. The crux of our proof is the following result by Alon et al.:

Theorem 9 ([1]). Given $k, n \geq 0$ and a set $V$ ofn elements, one can compute in time $2^{O(k)}|V| \log |V|$ a set of $l \in 2^{O(k)} \log |V| k$-coloring functions $c_{1}, \ldots c_{l}$ such that every set $S$ of $V$ of size $k$ is colorful for at least one $c_{i}(i \in[l])$.

To exploit this result when building incrementally a simple path from $x$ to $y$ we shall record for each coloring the subset of colors used so far, instead of recording the set of nodes visited. This reduces the number of combinations considered to $2^{O(k)} \log |V|$.

Proof of Theorem 8. Let $G, A_{L}, k$ be an instance of k-RSPQ. We compute $l$ k-coloring functions $c_{1}, \ldots c_{l}$ as stated in Theorem 9. Let $c$ be one of these functions. We will show how to decide if there is a colorful $L$-labeled path from $x$ to $y$ in $G$ for $c$. To this purpose, we define a function $f: V \times Q_{L} \times \mathcal{P}([k]) \rightarrow\{0,1\}$ such that $f(v, q, S)=1$ if and only if there exists a colorful path $p$ starting from $x$ that uses only colors of $S$ and such that $\Delta_{L}\left(i_{Q}, w\right)=q$ where $w$ is the label of $p$. The function can be computed by dynamic programming using the following equation.

- $f\left(x, i_{Q},\{c(x)\}\right)=1$

- $f(v, q, S)=1$ if there is a subset $S^{\prime} \subsetneq S$ such that $f\left(v, q, S^{\prime}\right)=1$;

- $f(v, q, S)=1$ if $c(v) \in S$ and there is a vertex $v^{\prime}$, a state $q^{\prime}$ and a label $a$ such that $f\left(v^{\prime}, q^{\prime}, S \backslash c(v)\right)=1,\left(v^{\prime}, a, v\right) \in E$ and $q \in \Delta_{L}\left(q^{\prime}, a\right)$

- $f(v, q, S)=0$ otherwise.

This function can be computed in time $O\left(2^{k} \cdot\left|A_{L}\right| \cdot|G|\right)$. We compute $f$ for every function $c_{i}, i \in[l]$ where $l \in 2^{O(k)} \log |V|$. Clearly there is a simple path of length at most $k$ from $x$ to $y$ if and only if there are $i \in[l], S \subseteq[k]$ and $q \in F_{L}$ such that $f(y, q, S)=1$ for coloring function $c_{i}$. Consequently, k-RSPQ can be solved in time $2^{O(k)}\left|A_{L}\right| \cdot|G| \cdot \log |G|$.

As a consequence of this theorem we get:

Corollary 3. para-RSPQ restricted to finite languages is FPT.

\subsection{Directed treewidth}

Directed treewidth is a notion introduced in [14]. It measures in some sense how close a digraph is to a directed acyclic graph and is based on the notion of arboreal decomposition. Johnson et al. [14] present a general method to design polynomial algorithms on graphs of bounded directed treewidth. Like most algorithms exploiting treewidth, this method leverages a dynamic programming approach on the decomposition tree. They apply this method to show that testing the 
existence of a Hamiltonian path is polynomial on such classes of graphs. Here, we extend this result to show that the regular simple path problem is also computable in polynomial time for the same classes.

It has been observed in the literature that RSPQ has polynomial combined complexity on two interesting classes of graphs: graphs of bounded treewidth [6], and DAGs [20]. The result for DAGs is immediate indeed, as every path in a DAG is simple. The next theorem generalizes both these two results.

Theorem 10. Let $L$ be a regular language, $k \geq 0$ and $\mathcal{G}$ be a class of $d b$-graphs with directed treewidth at most $k$. Then, $R S P Q(L)$ restricted to the class $\mathcal{G}$ is polynomial if an arboreal decomposition of the graph is given as input. Furthermore, it is also polynomial if $L$ is a part of the problem.

Proof. The proof is a straightforward adaptation of the proof proposed in [14] for the Hamiltonian Path problem. Since they use a dynamic approach, they consider a more general problem: given a digraph $G$ and a sequence of k tuples $\left(v_{i}, n_{i}, v_{i}^{\prime}\right)_{i \in[k]}$, are there $k$ disjoint simple paths $p_{1}, \ldots p_{k}$ such that $p_{i}$ is a path of size $n_{i}$ from $v_{i}$ to $v_{i}^{\prime}$ for every $i \in[k]$ ?

We extend the problem as follows: given a db-graph $G$, a regular language $L$ and a sequence of $\mathrm{k}$ tuples $\left(v_{i}, n_{i}, v_{i}^{\prime}, q_{i}\right.$, $\left.q_{i}^{\prime}\right)_{i \in[k]}$, are there $k$ words $w_{1}, \ldots w_{k}$ and $k$ disjoint simple paths $p_{1}, \ldots p_{k}$ such that $p_{i}$ is a $w_{i}$-labeled path of size $n_{i}$ from $v_{i}$ to $v_{i}^{\prime}$ and $\Delta_{L}\left(q_{i}, w_{i}\right)=q_{i}^{\prime}$ for every $i \in[k]$ ? Therefore, their proof can easily be adapted to this new problem.

\section{Future work}

We now pinpoint some directions for future work.

- As an extension of our work, we can consider context-free languages. It seems to be difficult to obtain useful results, since we can easily prove that distinguishing polynomial and NP-hard instances is undecidable if $\mathrm{P} \neq \mathrm{NP}$.

- What becomes tractable under restrictions to the graph such as planar digraphs or undirected graphs? Notice that both disjoint paths and even path problems are polynomial in these cases [16,21,26,28].

- Study the parametrized complexity of RSPQ for languages $L$ represented by NFAs, the parameter is the number of states of the NFA (or $L$ is represented by a regular expression and the parameter is the size of the regular expression). Corollary 3 proves that the complexity is FPT when $L$ is restricted to be finite.

\section{Declaration of competing interest}

The authors declare that they have no known compe5ng financial interests or personal rela5onships that could have appeared to influence the work reported in this paper.

\section{Acknowledgments}

The authors would like to thank the anonymous reviewers and Tina Trautner for their insightful comments on the manuscript.

\section{References}

[1] N. Alon, R. Yuster, U. Zwick, Color-coding, J. ACM 42 (4) (1995) 844-856.

[2] R. Angles, M. Arenas, P. Barceló, A. Hogan, J.L. Reutter, D. Vrgoc, Foundations of modern query languages for graph databases, ACM Comput. Surv. 50 (5) (2017) 68:1-68:40.

[3] M. Arenas, S. Conca, J. Pérez, Counting beyond a Yottabyte, or how SPARQL 1.1 property paths will prevent adoption of the standard, in: WWW, 2012, pp. 629-638.

[4] E.M. Arkin, C.H. Papadimitriou, M. Yannakakis, Modularity of cycles and paths in graphs, J. ACM 38 (2) (1991) $255-274$.

[5] G. Bagan, A. Bonifati, B. Groz, A trichotomy for regular simple path queries on graphs, in: Proceedings of the 32nd ACM SIGMOD-SIGACT-SIGAI Symposium on Principles of Database Systems, ACM, 2013, pp. 261-272.

[6] C.L. Barrett, R. Jacob, M.V. Marathe, Formal-language-constrained path problems, SIAM J. Comput. 30 (3) (2000) $809-837$.

[7] A. Bielefeldt, J. Gonsior, M. Krötzsch, Practical linked data access via SPARQL: the case of Wikidata, in: Proceedings of LDOW Workshop, 2018.

[8] A. Bonifati, W. Martens, T. Timm, An analytical study of large SPARQL query logs, VLDB J. 11 (2) (2017) 149-161.

[9] A. Bonifati, W. Martens, T. Timm, Navigating the maze of Wikidata query logs, in: The World Wide Web Conference, WWW 2019, San Francisco, CA, USA, May 13-17, 2019, 2019, pp. 127-138.

[10] M.R. Garey, D.S. Johnson, Computers and Intractability: A Guide to the Theory of NP-Completeness, W. H. Freeman, 1979.

[11] N. Immerman, Nondeterministic space is closed under complementation, SIAM J. Comput. 17 (5) (1988) $935-938$.

[12] N. Immerman, Descriptive Complexity, Springer, 1999.

[13] R. Jin, H. Hong, H. Wang, N. Ruan, Y. Xiang, Computing label-constraint reachability in graph databases, in: SIGMOD Conference, 2010 , pp. 123-134.

[14] T. Johnson, N. Robertson, P.D. Seymour, R. Thomas, Directed tree-width, J. Comb. Theory, Ser. B 82 (1) (2001) 138-154.

[15] A. Koschmieder, U. Leser, Regular path queries on large graphs, in: SSDBM, 2012, pp. 177-194.

[16] A.S. Lapaugh, C.H. Papadimitriou, The even-path problem for graphs and digraphs, Networks 14 (4) (1984) 507-513.

[17] U. Leser, A query language for biological networks, in: ECCB/JBI, 2005, p. 39

[18] K. Losemann, W. Martens, The complexity of regular expressions and property paths in SPARQL, ACM Trans. Database Syst. (TODS) 38 (4) (2013) 24.

[19] W. Martens, T. Trautner, Evaluation and enumeration problems for regular path queries, in: ICDT, in: LIPIcs, vol. 98, Schloss Dagstuhl - Leibniz-Zentrum fuer Informatik, 2018, pp. 19:1-19:21. 
[20] A.O. Mendelzon, P.T. Wood, Finding regular simple paths in graph databases, SIAM J. Comput. 24 (6) (1995) 1235-1258.

[21] Z.P. Nedev, Finding an even simple path in a directed planar graph, SIAM J. Comput. 29 (1999) 685-695.

[22] Z.P. Nedev, P.T. Wood, A polynomial-time algorithm for finding regular simple paths in outerplanar graphs, J. Algorithms 35 (2) (2000) 235-259.

[23] F. Olken, Graph data management for molecular biology, Omics. J. Integr. Biol. 7 (1) (2003) 75-78.

[24] C.H. Papadimitriou, Computational Complexity, Addison-Wesley, 1994.

[25] M. Perles, M. Rabin, E. Shamir, The theory of definite automata, IEEE Trans. Electron. Comput. EC-12 (3) (June 1963) 233-243.

[26] N. Robertson, P.D. Seymour, Graph minors XIII. The disjoint paths problem, J. Comb. Theory, Ser. B 63 (1) (1995) 65-110.

[27] W.L. Ruzzo, J. Simon, M. Tompa, Space-bounded hierarchies and probabilistic computations, J. Comput. Syst. Sci. 28 (2) (1984) $216-230$.

[28] A. Schrijver, Finding $k$ disjoint paths in a directed planar graph, SIAM J. Comput. 23 (4) (1994) 780-788.

[29] M.P. Schützenberger, On finite monoids having only trivial subgroups, Inf. Control 8 (2) (1965) 190-194.

[30] C.B. Ward, N.M. Wiegand, Complexity results on labeled shortest path problems from wireless routing metrics, Comput. Netw. 54 (2) (2010) 208-217. 